JOURNAL OF

SYMPLECTIC GEOMETRY

Volume 7, Number 4, 449-473, 2009

\title{
TOWARDS GENERALIZING SCHUBERT CALCULUS IN THE SYMPLECTIC CATEGORY
}

\author{
Rebecca F. Goldin and Susan Tolman
}

The main purpose of this article is to extend some of the ideas from Schubert calculus to the more general setting of Hamiltonian torus actions on compact symplectic manifolds with isolated fixed points. Given a generic component $\Psi$ of the moment map, which is a Morse function, we define a canonical class $\alpha_{p}$ in the equivariant cohomology of the manifold $M$ for each fixed point $p \in M$. When they exist, canonical classes form a natural basis of the equivariant cohomology of $M$; in particular, when $M$ is a flag variety, these classes are the equivariant Schubert classes. We show that the restriction of a canonical class $\alpha_{p}$ to a fixed point $q$ can be calculated by a rational function which depends only on the value of the moment map, and the restriction of other canonical classes to points of index exactly two higher. Therefore, the structure constants can be calculated by a similar rational function. Our restriction formula is manifestly positive in many cases, including when $M$ is a flag manifold. Finally, we prove the existence of integral canonical classes in the case that $M$ is a GKM space (after Goresky, Kottwitz and MacPherson) and $\Psi$ is index increasing. In this case, our restriction formula specializes to an easily computable rational sum which depends only on the GKM graph.

\section{Contents}

1. Introduction

2. Canonical classes $\quad 455$

3. Proof of Theorem $1.2 \quad 460$

4. The GKM case $\quad 464$

5. Examples $\quad 470$

References $\quad 472$ 


\section{Introduction}

Let $T$ be a compact torus with Lie algebra $\mathfrak{t}$ and lattice $\ell \subset \mathfrak{t}$. Suppose that $T$ acts on a compact symplectic manifold $(M, \omega)$ with isolated fixed points and moment map $\Phi: M \rightarrow \mathfrak{t}^{*}$, where $\mathfrak{t}^{*}$ is dual to $\mathfrak{t}$. Then

$$
\iota^{\prime} x_{\xi} \omega=-\mathrm{d} \Phi^{\xi} \quad \forall \xi \in \mathfrak{t},
$$

where $X_{\xi}$ denotes the vector field on $M$ generated by the action and $\Phi^{\xi}$ : $M \rightarrow \mathbb{R}$ is defined by $\Phi^{\xi}(x)=\langle\Phi(x), \xi\rangle$. Here, $\langle\cdot, \cdot\rangle$ is the natural pairing between $\mathfrak{t}^{*}$ and $\mathfrak{t}$.

If $\xi \in \mathfrak{t}$ is generic, that is, if $\langle\eta, \xi\rangle \neq 0$ for each weight $\eta \in \ell^{*} \subset \mathfrak{t}^{*}$ in the symplectic representation $T_{p} M$ for every $p$ in the fixed set $M^{\top}$, then $\Psi=\Phi^{\xi}: M \rightarrow \mathbb{R}$ is a Morse function with critical set $M^{\top}$. Given $p \in M^{\top}$, the negative tangent bundle $v^{-}(p)$ is a representation with no fixed subbundle. Hence, the index of $\Psi$ at $p$ is even; let $\lambda(p)$ denote half the index of $\Psi$ at $p$. The individual weights of this representation are well defined and non-zero; our convention for the moment map implies that these weights are exactly the positive weights of the $T$ action on $T_{p} M$, that is, the weights $\eta$ such that $\langle\eta, \xi\rangle>0$. Let $\Lambda_{p}^{-}$denote the product of these weights. (Conversely, the weights in the positive tangent bundle are the negative weights of the $T$ action on $T_{p} M$.) Finally, for all $q \in M^{\top}$ the inclusion $\mathrm{q} \hookrightarrow M$ induces a map $\mathrm{H}_{\mathrm{T}}^{*}(\mathrm{M}) \rightarrow \mathrm{H}_{\mathrm{T}}^{*}(\mathrm{q})$ in equivariant cohomology; let $\alpha(q)$ denote the image of a class $\alpha \in \mathrm{H}_{\mathrm{T}}^{*}(M)$ under this map.

Definition 1.1. Let a torus $T$ act on a compact symplectic manifold $(M, \omega)$ with isolated fixed points and moment map $\Phi: M \rightarrow \mathfrak{t}^{*}$. Let $\Psi=\Phi^{\xi}$ : $M \rightarrow \mathbb{R}$ be a generic component of the moment map. A cohomology class $\alpha_{\mathrm{p}} \in \mathrm{H}_{\mathrm{T}}^{2 \lambda(p)}(\mathrm{M} ; \mathbb{Q})$ is the canonical class at a fixed point $\mathrm{p}$ with respect to $\Psi$ if

(1) $\alpha_{p}(p)=\Lambda_{p}^{-}$

(2) $\alpha_{p}(q)=0$ for all $q \in M^{\top} \backslash\{p\}$ such that $\lambda(q) \leq \lambda(p) .{ }^{1}$

Moreover, we say the canonical class $\alpha_{p}$ is integral if $\alpha_{p} \in H_{T}^{2 \lambda(p)}(M ; \mathbb{Z}) .^{2}$

We cannot always find canonical classes; see Example 2.2. However, each canonical class is unique and can be thought of as an equivariant Poincaré dual to the closure of the stable manifold. If $\alpha_{p}$ exists for all $p \in M^{\top}$, then $\left\{\alpha_{p}\right\}$ forms a basis of $H_{T}^{*}(M)$ as a module over $H^{*}(B T)$. Since the fixed set is isolated, the natural restriction map $H_{T}^{*}(M ; \mathbb{Z}) \rightarrow H^{*}(M ; \mathbb{Z})$ is surjective; under this map, the canonical classes also define a basis for the

\footnotetext{
${ }^{1}$ Note that (2) is stronger than the frequently encountered condition that $\alpha_{\mathrm{p}}(\mathrm{q})=0$ for all $q \in M^{\top} \backslash\{p\}$ such that $\Psi(q) \leq \Psi(p)$. See Lemmas 2.5 and 2.8 .

${ }^{2}$ Since the fixed points are isolated, $\mathrm{H}_{\mathrm{T}}^{*}(M ; \mathbb{Z})$ is torsion free; see Lemma 2.5. Therefore, we can naturally identify $\mathrm{H}_{\mathrm{T}}^{*}(M ; \mathbb{Z})$ with a subgroup of $\mathrm{H}_{\mathrm{T}}^{*}(M ; \mathbb{Q})$.
} 
ordinary cohomology $H^{*}(M)$. In the case that $M=G / B$, where $G$ is a complex semi-simple Lie group (of any type) and B is a Borel subgroup, the equivariant Schubert classes are canonical classes. Under the map to ordinary cohomology, they are exactly the Poincaré duals to Schubert varieties in ordinary cohomology. Hence, our work is a direct generalization of that setting.

This paper is concerned with a new formula for how to restrict canonical cohomology classes to fixed points. Since the fixed points are isolated, the inclusion of the fixed point set $M^{\top}$ into $M$ induces an injection $\mathrm{H}_{\mathrm{T}}^{*}(M ; \mathbb{Z}) \rightarrow$ $\mathrm{H}_{\mathrm{T}}^{*}\left(M^{\top} ; \mathbb{Z}\right)$, where the latter ring is a direct sum of polynomials rings. Thus each cohomology class on $M$ may be described by an integral polynomial associated to each fixed point. Once the restriction of canonical classes is known at each fixed point, one can easily derive a formula for the structure constants in the (equivariant) cohomology ring. (See $[\mathbf{G Z}]$.) Recall that the structure constants for $\mathrm{H}_{\mathrm{T}}^{*}(M)$ are the set $\mathrm{c}_{\mathrm{pq}}^{\mathrm{r}} \in \mathrm{H}_{\mathrm{T}}^{*}(M)$ given by

$$
\alpha_{p} \alpha_{q}=\sum_{r \in M^{T}} c_{p q}^{r} \alpha_{r}
$$

Conversely, the structure constants also provide a formula for the restrictions.

Our formulas have some echoes in the literature; Billey [Bi] found a different manifestly positive formula for the restriction of equivariant Schubert classes when $M=G / B$. Guillemin and Zara $[\mathbf{G Z}]$ found a non-positive path formula for the restrictions in the case of GKM graphs, which we discuss in more detail below.

Our main contribution in this article can be seen as an inductive formula for the restriction of canonical classes to fixed points; we prove this in Section 3. The formula depends on only the values of the moment map and $\alpha_{r}\left(r^{\prime}\right)$, where $r$ and $r^{\prime}$ are fixed points whose indices differ by 2 .

Given a directed graph with vertex set $\mathrm{V}$ and edge set $\mathrm{E} \subset \mathrm{V} \times \mathrm{V}$, a path from a vertex $p$ to a vertex $q$ is a $(k+1)$-tuple $\mathbf{r}=\left(r_{0}, \ldots, r_{k}\right) \in V^{k+1}$ so that $r_{0}=p, r_{k}=q$, and $\left(r_{i-1}, r_{i}\right) \in E$ for all $1 \leq i \leq k$; let $|\mathbf{r}|=k$ denote the length of $\mathbf{r}$.

Theorem 1.2. Let a torus $\mathrm{T}$ act on a compact symplectic manifold $(\mathrm{M}, \boldsymbol{\omega})$ with isolated fixed points and moment map $\Phi: M \rightarrow \mathfrak{t}^{*}$. Let $\Psi=\Phi^{\xi}$ be a generic component of the moment map. Assume that there exists a canonical class $\alpha_{\mathrm{p}} \in \mathrm{H}_{\mathrm{T}}^{2 \lambda(\mathrm{p})}(\mathrm{M} ; \mathbb{Q})$ for all $\mathrm{p} \in \mathrm{M}^{\mathrm{T}}$.

Define an oriented graph with vertex set $\mathrm{V}=\mathrm{M}^{\top}$ and edge set

$$
E=\left\{\left(r, r^{\prime}\right) \in M^{\top} \times M^{\top} \mid \lambda\left(r^{\prime}\right)-\lambda(r)=1 \text { and } \alpha_{r}\left(r^{\prime}\right) \neq 0\right\}
$$


Given $\mathrm{p}$ and $\mathrm{q}$ in $\mathrm{M}^{\mathrm{\top}}$, let $\Sigma_{\mathrm{p}}^{\mathrm{q}}$ denote the set of paths from $\mathrm{p}$ to $\mathrm{q}$ in $(\mathrm{V}, \mathrm{E})$; then

$$
\alpha_{p}(\mathbf{q})=\Lambda_{\mathbf{q}}^{-} \sum_{\mathbf{r} \in \Sigma_{p}^{q}} \prod_{i=1}^{|\mathbf{r}|} \frac{\Phi\left(r_{i}\right)-\Phi\left(r_{i-1}\right)}{\Phi(\mathbf{q})-\Phi\left(r_{i-1}\right)} \frac{\alpha_{r_{i-1}}\left(r_{i}\right)}{\Lambda_{r_{i}}^{-}}
$$

Remark 1.3 (Positivity). We say that $\alpha \in S\left(t^{*}\right)$ is positive if $\alpha(\xi)>0$ and negative if $\alpha(\xi)<0$. In some cases, the restriction $\alpha_{p}(q)$ is itself negative; see Example 5.2. A fortiori, in these examples some of the summands in (1.1) are negative. However, whenever $\alpha_{p}(q) \geq 0$ for all $p$ and $q \in M^{\top}$ such that $\lambda(q)=\lambda(p)+1$, our formula is manifestly positive, in the sense that each summand is positive. To see this, note that $\Lambda_{\mathrm{q}}^{-}$and $\Lambda_{\mathrm{r}_{\mathrm{i}}}^{-}$are positive by definition, $\Phi\left(r_{i}\right)-\Phi\left(r_{i-1}\right)$ and $\Phi(q)-\Phi\left(r_{i-1}\right)$ are positive by Corollary 2.9 , and $\alpha_{r_{i-1}}\left(r_{i}\right)$ is positive by assumption.

For example, for flag varieties $G / B$ of semi-simple Lie groups the canonical classes are Schubert classes; see [BGG]. In this case, the restriction $\alpha_{p}(q)$ is positive for all $p$ and $q$ by $[\mathbf{B i}]$. Alternatively, it is very easy to check this directly when $\lambda(q)=\lambda(p)+1$; see Section 5 for the case $G=\operatorname{Sl}(n, \mathbb{C})$ and $[\mathbf{S T}]$ for the general case.

Corollary 1.4. Consider the situation described in Theorem 1.2. If there is no path in $(\mathrm{V}, \mathrm{E})$ from a fixed point $\mathrm{p}$ to a fixed point $\mathrm{q}$, then $\alpha_{\mathrm{p}}(\mathrm{q})=0$. Moreover, if $\alpha_{p}(q) \geq 0$ for all $p$ and $q$ in $M^{\top}$ such that $\lambda(q)=\lambda(p)+1$, then $\alpha_{p}(q) \geq 0$ for all $p$ and $q$ in $M^{\top}$ and $\alpha_{p}(q)>0$ exactly if there is at least one path from $\mathrm{p}$ to $\mathrm{q}$.

We now restrict our attention to an important special case where it is especially easy to make these calculations: GKM spaces. Let a torus T act on a compact symplectic manifold $(M, \omega)$ with moment map $\Phi: M \rightarrow \mathfrak{t}^{*}$. We say that $(M, \omega, \Phi)$ is a GKM space if $M$ has isolated fixed points and if, for every codimension one subgroup $\mathrm{K} \subset \mathrm{T}$, every connected component of the fixed submanifold $\mathrm{M}^{\mathrm{K}}$ has dimension two or less.

Definition 1.5. Let $(M, \omega, \Phi)$ be a GKM space. We define the GKM graph to be the labelled directed graph $\left(\mathrm{V}, \mathrm{E}_{\mathrm{GKM}}\right)$ given as follows. The vertex set $V$ is the fixed set $M^{\top}$; we label each $p \in M^{\top}$ by its moment image $\Phi(p) \in \mathfrak{t}^{*}$. The edge set $E_{G K M}$ consists of pairs of distinct points $(p, q) \in V \times V$ such that there exists a codimension one subgroup $\mathrm{K} \subset \mathrm{T}$ so that $p$ and $q$ are contained in the same component $N$ of $M^{K}$. We label the edge $(p, q)$ by the weight $\eta(p, q) \in \ell^{*}$ associated to the representation of $T$ on $\mathrm{T}_{\mathrm{q}} \mathrm{N} \simeq \mathbb{C}$.

Let $\Psi=\Phi^{\xi}$ be a generic component of the moment map. Note that $\lambda(p)$ is the number of edges $(r, p) \in E_{G K M}$ such that $\Psi(r)<\Psi(p)$; moreover, $\Lambda_{p}^{-}=\prod \eta(r, p)$, where the product is over all such edges. We say that 
$\Psi$ is index increasing if $\Psi(p)<\Psi(q)$ implies that $\lambda(p)<\lambda(q)$ for all $(p, q) \in E_{G K M}$. See Remark 4.3.

Given any weight $\eta \in \ell^{*}$, the projection which takes $X \in \mathfrak{t}^{*}$ to $X-\frac{\langle X, \xi\rangle}{\langle\eta, \xi\rangle} \eta \in$ $\xi^{\perp} \subset \mathfrak{t}^{*}$ naturally induces a endomorphism $\rho_{\eta}$ of $S\left(\mathfrak{t}^{*}\right)$, the symmetric algebra on $t^{*}$. Since $M$ is a GKM space, the weights at each fixed point are pairwise linearly independent; hence, $\rho_{\eta\left(r, r^{\prime}\right)}\left(\Lambda_{r}^{-}\right) \neq 0$ and $\rho_{\eta\left(r, r^{\prime}\right)}\left(\frac{\Lambda_{r^{\prime}}^{-}}{\eta\left(r, r^{\prime}\right)}\right) \neq 0$ for all $\left(r, r^{\prime}\right) \in E_{G K M}$. Following $[\mathbf{G Z}]$, we define

$$
\Theta\left(r, r^{\prime}\right)=\frac{\rho_{\eta\left(r, r^{\prime}\right)}\left(\Lambda_{r}^{-}\right)}{\rho_{\eta\left(r, r^{\prime}\right)}\left(\frac{\Lambda_{r}^{-}}{\eta\left(r, r^{\prime}\right)}\right)} \in S\left(t^{*}\right)_{0} \quad \forall\left(r, r^{\prime}\right) \in E_{G K M},
$$

where $S\left(\mathfrak{t}^{*}\right)_{0}$ is the field of fractions of $S\left(\mathfrak{t}^{*}\right)$.

Theorem 1.6. Let $(M, \omega, \Phi)$ be GKM space. Let $\Psi=\Phi^{\xi}$ be a generic component of the moment map; assume that $\Psi$ is index increasing. Define an oriented graph with vertex set $\mathrm{V}=\mathrm{M}^{\top}$ and edge set

$$
\mathrm{E}=\left\{\left(\mathrm{r}, \mathrm{r}^{\prime}\right) \in \mathrm{E}_{\mathrm{GKM}} \mid \lambda\left(\mathrm{r}^{\prime}\right)-\lambda(\mathrm{r})=1\right\},
$$

where $\left(\mathrm{V}, \mathrm{E}_{\mathrm{GKM}}\right)$ is the GKM graph associated to $\mathrm{M}$. Then

- there exists a canonical class $\alpha_{\mathrm{p}} \in \mathrm{H}_{\mathrm{T}}^{\lambda(\mathrm{p})}(\mathrm{M} ; \mathbb{Z})$ for all $\mathrm{p} \in \mathrm{M}^{\top}$,

- given $\mathrm{p}$ and $\mathrm{q}$ in $\mathrm{M}^{\mathrm{\top}}$, let $\Sigma_{\mathrm{p}}^{\mathrm{q}}$ denote the set of paths from $\mathrm{p}$ to $\mathrm{q}$ in $(\mathrm{V}, \mathrm{E})$, then

$$
\alpha_{p}(q)=\Lambda_{q}^{-} \sum_{r \in \Sigma_{p}^{q}} \prod_{i=1}^{|r|} \frac{\Phi\left(r_{i}\right)-\Phi\left(r_{i-1}\right)}{\Phi(q)-\Phi\left(r_{i-1}\right)} \frac{\Theta\left(r_{i-1}, r_{i}\right)}{\eta\left(r_{i-1}, r_{i}\right)} .
$$

- $\Theta\left(r, r^{\prime}\right) \in \mathbb{Z} \backslash\{0\}$ for all $\left(r, r^{\prime}\right) \in E$.

Remark 1.7. A straightforward calculation shows that, since $\Theta\left(r, r^{\prime}\right)$ is an integer,

$$
\frac{\Lambda_{r^{\prime}}^{-}}{\eta\left(r, r^{\prime}\right)} \Theta\left(r, r^{\prime}\right)=\Lambda_{r}^{-} \bmod \eta\left(r, r^{\prime}\right)
$$

Moreover, since $\frac{\Lambda_{r^{\prime}}^{-}}{\eta\left(r, r^{\prime}\right)}$ is not a multiple of $\eta\left(r, r^{\prime}\right)$, equation (1.4) has a unique solution and so provides an alternative definition of $\Theta$.

Remark 1.8. In fact, since $\Phi\left(r^{\prime}\right)-\Phi(r)$ is a positive multiple of $\eta\left(r, r^{\prime}\right)$ for all $\left(r, r^{\prime}\right) \in E_{G K M}$, formula (1.3) is a manifestly positive exactly if $\Theta\left(r, r^{\prime}\right)>$ 0 for all $\left(r, r^{\prime}\right) \in E_{G K M}$; cf. Remark 1.3. However, $\Theta\left(r, r^{\prime}\right)$ is not always positive; see Example 5.2.

Example 1.9. $e_{1}, \ldots, e_{n+1}$ denote the standard basis for $\mathbb{R}^{n+1}$, and $x_{1}, \ldots, x_{n+1}$ denote the dual basis for $\left(\mathbb{R}^{n+1}\right)^{*}$. Let $S_{\Delta}^{1} \subset\left(S^{1}\right)^{n+1}$ be 
the diagonal circle, and $T=\left(S^{1}\right)^{n+1} / S_{\Delta}^{1}$ the quotient torus. The standard action of $\left(S^{1}\right)^{n+1}$ on $\mathbb{C}^{n+1}$ induces a symplectic action of $T$ on complex projective space $\left(\mathbb{C P}^{n}, \omega\right)$ with moment map $\Phi: \mathbb{C P}^{n} \rightarrow \mathfrak{t}^{*}$, where $\mathfrak{t}^{*}=\left\{\sum a_{i} x_{i} \in\left(\mathbb{R}^{n+1}\right)^{*} \mid \sum a_{i}=0\right\}$, and

$$
\Phi\left(\left[z_{1}: \cdots: z_{n+1}\right]\right)=\sum_{i=1}^{n+1}\left(\frac{1}{n+1}-\frac{\left|z_{i}\right|^{2}}{\sum_{j}\left|z_{j}\right|^{2}}\right) x_{i} .
$$

It is straightforward to check that $\left(\mathbb{C P}^{n}, \omega, \Phi\right)$ is a $\mathrm{GKM}$ space, and that the associated GKM graph is the complete directed graph on $n+1$ vertices $p_{1}, \ldots, p_{n+1}$, where $p_{i}=\left[e_{i} \otimes \mathbb{C}\right]$. Moreover, $\Phi\left(p_{i}\right)=\frac{1}{n+1} \sum_{j} x_{j}-x_{i}$ for all $i$, and $\eta\left(p_{i}, p_{j}\right)=x_{i}-x_{j}$ for all $i \neq j$.

Let $\Psi=\Phi^{\xi}$, where $\xi=-(0,1,2, \ldots, n)$. Then $\Psi\left(p_{i}\right)-\Psi\left(p_{j}\right)=$ $\left\langle-x_{i}+x_{j}, \xi\right\rangle=i-j$ is positive exactly if $j<i$, and so $\Lambda_{p_{i}}^{-}=\prod_{j<i}\left(x_{j}-\right.$ $\left.x_{i}\right)$ and $\lambda\left(p_{i}\right)=i-1$ for all $i$. Therefore, $\Psi$ is index increasing and $E=\left\{\left(p_{1}, p_{2}\right), \ldots,\left(p_{n}, p_{n+1}\right)\right\}$. In particular, there is exactly one path $\left(p_{i}, p_{i+1}, \ldots, p_{j}\right)$ from $p_{i}$ to $p_{j}$ in $E$ if $i \leq j$; otherwise, there is none. Finally, since $\rho_{x_{l-1}-x_{l}}\left(x_{l}\right)=\rho_{x_{l-1}-x_{l}}\left(x_{l-1}\right)$,

$$
\begin{aligned}
\Theta\left(p_{l-1}, p_{l}\right) & =\frac{\rho_{\eta\left(p_{l-1}, p_{l}\right)}\left(\Lambda_{p_{l-1}}^{-}\right)}{\rho_{\eta\left(p_{l-1}, p_{l}\right)}\left(\frac{\Lambda_{p_{l}}^{-}}{\eta\left(p_{l-1}, p_{l}\right)}\right)} \\
& =\frac{\rho_{x_{l-1}-x_{l}}\left(\prod_{m<l-1}\left(x_{m}-x_{l-1}\right)\right)}{\rho_{x_{l-1}-x_{l}}\left(\prod_{m<l-1}\left(x_{m}-x_{l}\right)\right)}=1 \quad \forall l .
\end{aligned}
$$

Thus, by Theorem 1.6,

$$
\begin{aligned}
\alpha_{p_{i}}\left(p_{j}\right) & =\Lambda_{p_{j}}^{-} \prod_{l=i+1}^{j} \frac{\Phi\left(p_{l}\right)-\Phi\left(p_{l-1}\right)}{\Phi\left(p_{j}\right)-\Phi\left(p_{l-1}\right)} \frac{\Theta\left(p_{l-1}, p_{l}\right)}{\eta\left(p_{l-1}, p_{l}\right)} \\
& =\prod_{l=1}^{j-1}\left(x_{l}-x_{j}\right) \prod_{l=i+1}^{j} \frac{x_{l-1}-x_{l}}{x_{l-1}-x_{j}} \frac{1}{x_{l-1}-x_{l}} \\
& =\prod_{l=1}^{j-1}\left(x_{l}-x_{j}\right) \prod_{l=i}^{j-1} \frac{1}{x_{l}-x_{j}}=\prod_{l=1}^{i-1}\left(x_{l}-x_{j}\right) .
\end{aligned}
$$

Remark 1.10. Guillemin and Zara also give a formula for $\alpha_{\mathfrak{p}}(\mathbf{q})$ for GKM spaces as a sum over paths in $[\mathbf{G Z}]$. In fact, their formula is identical to ours in the case that $\lambda(q)-\lambda(p)=1$, and also works in a slightly broader context. However, in general the formulas are quite different. For example, their formula for $\alpha_{p}(q)$ includes a contribution for each path $r$ from $p$ to $q$ in $\left(V, E_{G K M}\right)$ such that $\Psi\left(r_{i}\right)>\Psi\left(r_{i-1}\right)$ for each edge $\left(r_{i-1}, r_{i}\right)$; our formula only includes a contribution from a subset of such paths - those such that $\lambda\left(r_{i}\right)=\lambda\left(r_{i-1}\right)+1$. In practice, this means that we sum over many fewer 
paths. For example, if $M=\mathbb{C} p^{n}$ their formula for $\alpha_{p}(q)$ contains $2^{\lambda(q)-\lambda(p)-1}$ terms, whereas ours contains just one term; see Example 1.9. Moreover, their formula is almost never manifestly positive, in the sense described above.

In [Kn99], Knutson gives a positive formula for the DuistermaatHeckman measure of a torus action on a smooth algebraic variety ${ }^{3}$ with an invariant Palais-Smale metric, and suggests a technique for computing the Duistermaat-Heckman measure of certain subvarieties. In fact, in the case that $M$ is an algebraic variety and there exists an invariant PalaisSmale metric, it is possible to use the results of [Kn99] to give an alternate proof of Theorem 1.2. We hope to do this in our next paper; we also plan to use Theorem 1.2 to extend his formula for the Duistermaat-Heckman measure to the non-algebraic case. However, in this greater generality, the summands in the formula are not always positive. This occurs, for example, in the manifold considered in Example 5.2. After we initially announced these results, Knutson showed that he could extend his results in [Kn99] by dropping the condition that there exist an invariant Palais-Smale metric; see $[$ Kn10].

Finally, several techniques have recently been discovered which use the ideas in this paper to find a positive integral formula in certain important cases, including when $M$ is a flag manifold ([ST, Za $]$ ).

We would like to thank Victor Guillemin, whose questions inspired this project. We would also like to thank Sara Billey, Allen Knutson, Catalin Zara, and Silvia Sabatini for many helpful discussions.

\section{Canonical classes}

In this section, we demonstrate some properties of canonical classes. In particular, we show that if they exist then they form a natural basis for $\mathrm{H}_{\mathrm{T}}^{*}(M ; \mathbb{Z})$ as a $\mathrm{H}^{*}(\mathrm{BT} ; \mathbb{Z})$ module. Additionally, they do exist in a number of important cases.

For this purpose, it is natural to work in a slightly more general context. Let a torus $T$ act on a compact oriented manifold $M$ with isolated fixed points. An invariant Morse function $\Psi: M \rightarrow \mathbb{R}$ is a formal moment map $^{4}$ if the critical set of $\Psi$ is exactly the fixed point set $M^{\top}$. As we saw in the introduction, if $M$ is symplectic and the action is Hamiltonian, then any generic component of the moment map is a formal moment map. The cohomological properties of symplectic manifolds with Hamiltonian actions described in the introduction continue to hold in the more general case of formal moment maps; see Appendix G of $[\mathbf{G G K}$. In particular, the restriction map from $H_{T}^{*}(M ; \mathbb{Z})$ to $H_{T}^{*}\left(M^{\top} ; \mathbb{Z}\right)$ is injective in this case.

\footnotetext{
${ }^{3}$ The restriction to the algebraic category is only implicit.

${ }^{4}$ In $[\mathbf{G G K}]$, these are called "non-degenerate abstract moment maps."
} 
Let $p$ be a critical point for a formal moment map $\Psi: M \rightarrow \mathbb{R}$. Since $v^{-}(p)$ is a real representation with no fixed subbundle, the index of $\Psi$ at $p$ is even; let $\lambda(p)$ denote half the index of $\Psi$ at $p$. The signs of the individual weights of this representation are not well defined. However, if we fix an orientation on the negative normal bundle $v^{-}(p)$ then the product of the weights — which we will denote $\Lambda_{p}^{-}$— is well defined.

Definition 2.1. Let a torus $T$ act on a compact oriented manifold $M$ with isolated fixed points, and let $\Psi: M \rightarrow \mathbb{R}$ be a formal moment map. We say that a cohomology class $\alpha_{p} \in H_{T}^{2 \lambda(p)}(M ; \mathbb{Q}$ ) is a canonical class (with respect to $\Psi)$ at a fixed point $p$ if there exists an orientation on $v^{-}(p)$ such that

(1) $\alpha_{p}(p)=\Lambda_{p}^{-}$and

(2) $\alpha_{p}(q)=0$ for all $q \in M^{\top} \backslash\{p\}$ such that $\lambda(q) \leq \lambda(p)$.

Moreover, the canonical class is integral if $\alpha_{\mathrm{p}} \in \mathrm{H}_{\mathrm{T}}^{2 \lambda(\mathrm{p})}(M ; \mathbb{Z})$.

Canonical classes do not always exist.

Example 2.2. Let the torus $T=\left(S^{1}\right)^{2}$ act on $\mathbb{C P}^{2}$ by

$$
\left(\mathrm{t}_{1}, \mathrm{t}_{2}\right)\left[z_{1}: z_{2}: z_{3}\right]=\left[\mathrm{t}_{1} z_{1}: \mathrm{t}_{2} z_{2}: z_{3}\right] \text {. }
$$

Let $M$ be the blow-up of $\mathbb{C P}^{2}$ at $[0: 0: 1]$, and let $\Phi: M \rightarrow \mathbb{R}^{2}$ be the moment map for the induced $T$ action. Let $\Psi=\Phi^{\xi}$, where $\xi=(1,-1) \in$ $\mathbb{R}^{2}$. Label the four fixed points $p_{1}, \ldots, p_{4}$ so that $\Psi\left(p_{1}\right)<\cdots<\Psi\left(p_{4}\right)$. There exists a basis $\gamma_{1}, \ldots, \gamma_{4}$ for $\mathrm{H}_{\mathrm{T}}^{*}(M ; \mathbb{Z})$ as a $\mathrm{H}^{*}(\mathrm{BT} ; \mathbb{Z})$ module so that $\gamma_{i}\left(p_{j}\right)=0 \forall j<i$ and

$$
\begin{gathered}
\gamma_{1}\left(p_{1}\right)=\gamma_{1}\left(p_{2}\right)=\gamma_{1}\left(p_{3}\right)=\gamma_{1}\left(p_{4}\right)=1 ; \quad \gamma_{2}\left(p_{2}\right)=\gamma_{2}\left(p_{3}\right)=x_{1} ; \\
\gamma_{2}\left(p_{4}\right)=x_{1}-x_{2} ; \quad \gamma_{3}\left(p_{3}\right)=\gamma_{3}\left(p_{4}\right)=x_{1}-x_{2} ; \quad \text { and } \quad \gamma_{4}\left(p_{4}\right)=-x_{2}\left(x_{1}-x_{2}\right) .
\end{gathered}
$$

A straightforward calculation shows that there is no canonical class for $p_{2}$. (Although this example is a GKM space, it is consistent with Theorem 1.6 because $\Psi$ is not index increasing.)

However, if canonical classes do exist, they give a natural basis for $\mathrm{H}_{\mathrm{T}}^{*}(M)$.

Proposition 2.3. Let a torus $\mathrm{T}$ act on a compact oriented manifold $\mathrm{M}$ with isolated fixed points, and let $\Psi: M \rightarrow \mathbb{R}$ be a formal moment map. Fix an orientation on $v^{-}(p)$ for all $p \in M^{\top}$. If there exists a canonical class $\alpha_{\mathrm{p}} \in \mathrm{H}_{\mathrm{T}}^{*}(\mathrm{M} ; \mathbb{Q})$ for all $\mathrm{p} \in \mathrm{M}^{\top}$, then the classes $\left\{\alpha_{\mathrm{p}}\right\}_{\mathrm{p} \in \mathrm{M}^{\top}}$ are a natural basis for $\mathrm{H}_{\mathrm{T}}^{*}(M ; \mathbb{Q})$ as a module over $\mathrm{H}^{*}(\mathrm{BT} ; \mathbb{Q})$. Moreover, if the canonical classes are integral then they are a natural basis for $\mathrm{H}_{\mathrm{T}}^{*}(\mathrm{M} ; \mathbb{Z})$ as a module over $\mathrm{H}^{*}(\mathrm{BT} ; \mathbb{Z})$.

Proof. This is an immediate consequence of Lemmas 2.5, 2.7, and 2.8 below. 
Remark 2.4. This basis does not depend very sensitively on the choices that we have made; it only depends on the $\Lambda_{p}^{-}$'s at each fixed point. For example, let a torus $T$ act on a compact symplectic manifold $(M, \omega)$ with isolated fixed points and moment map $\Phi: M \rightarrow \mathfrak{t}^{*}$. Let $\Psi=\Phi^{\xi}$ be a generic component of the moment map. Let $\alpha_{\mathrm{p}} \in \mathrm{H}_{\mathrm{T}}^{2 \lambda(\mathrm{p})}(M ; \mathbb{Z})$ be a canonical class at $p$ for $\Psi$. If $\Psi^{\prime}=\Phi^{\xi^{\prime}}$ is another generic component, then $\alpha_{p}$ is also a canonical class for $\Psi^{\prime}$, as long as

$$
\langle\xi, \eta\rangle>0 \leftrightarrow\left\langle\xi^{\prime}, \eta\right\rangle>0 \quad \forall \eta \in \Pi_{p} \quad \text { and } p \in M^{\top}
$$

where $\Pi_{p}$ denotes the set of weights at $p$. Similarly, if $\omega^{\prime} \in \Omega^{2}(M)$ is another invariant symplectic form with moment map $\Phi^{\prime}: M \rightarrow \mathfrak{t}$, then $\alpha_{\mathrm{p}}$ is also a canonical class for $\Psi^{\prime}=\left(\Phi^{\prime}\right)^{\xi}$ as long as $\omega$ and $\omega^{\prime}$ are deformation equivalent.

The following lemma is a key ingredient in our proof that canonical classes exist in certain cases. In particular, the existence of these closely related classes is guaranteed by straightforward Morse theoretic arguments.

Lemma 2.5 (Kirwan). Let a torus $\mathrm{T}$ act on a compact oriented manifold $M$ with isolated fixed points, and let $\Psi: M \rightarrow \mathbb{R}$ be a formal moment map. For every fixed point $p$ and for each orientation on $v^{-}(p)$, there exists an integral cohomology class $\gamma_{\mathrm{p}} \in \mathrm{H}_{\mathrm{T}}^{2 \lambda(\mathrm{p})}(\mathrm{M} ; \mathbb{Z})$ so that

(1) $\gamma_{\mathrm{p}}(\mathrm{p})=\Lambda_{\mathrm{p}}^{-}$, and

$\left(2^{\prime}\right) \gamma_{p}(q)=0$ for every $q \in M^{\top} \backslash\{p\}$ such that $\Psi(q) \leq \Psi(p)$.

Moreover, for any such classes, the $\left\{\gamma_{\mathrm{p}}\right\}_{\mathrm{p} \in \mathrm{M}^{\top}}$ are a basis for $\mathrm{H}_{\mathrm{T}}^{*}(\mathrm{M} ; \mathbb{Z})$ as a module over $\mathrm{H}^{*}(\mathrm{BT} ; \mathbb{Z})$.

Kirwan proved this result for rational cohomology classes on compact symplectic manifolds with Hamiltonian actions [Ki] (see also [TW]). The proof generalizes easily to the case of formal moment maps, and to integral cohomology when the fixed points are isolated.

Corollary 2.6. Let a torus $\mathrm{T}$ act on a compact oriented manifold $\mathrm{M}$ with isolated fixed points, and let $\Psi: M \rightarrow \mathbb{R}$ be a formal moment map. Given a point $\mathrm{p} \in \mathrm{M}^{\top}$ and a class $\beta \in \mathrm{H}_{\mathrm{T}}^{2 \mathrm{i}}(\mathrm{M} ; \mathbb{Q})$ such that $\beta(\mathrm{q})=0$ whenever $\mathrm{q} \in M^{\top}$ satisfies $\Psi(\mathrm{q})<\Psi(\mathrm{p})$,

- the restriction $\beta(p)=x \Lambda_{\mathrm{p}}^{-}$, where $x \in \mathrm{H}^{*}(\mathrm{BT} ; \mathbb{Q})$,

- in particular, if $\lambda(p)>i$ then $\beta(p)=0$, and

- if $\beta \in \mathrm{H}_{\mathrm{T}}^{*}(M ; \mathbb{Z})$ is integral then $\beta(\mathrm{p})=x \Lambda_{\mathrm{p}}^{-}$, where $\mathrm{x} \in \mathrm{H}^{*}(\mathrm{BT} ; \mathbb{Z})$ is integral.

Proof. By Lemma 2.5 for each $\mathrm{q} \in \mathrm{M}^{\top}$, we can fix an orientation on $v^{-}(\mathrm{q})$ and choose a class $\gamma_{\mathrm{q}} \in \mathrm{H}_{\mathrm{T}}^{*}(M ; \mathbb{Z})$ which satisfies properties $(1)$ and $\left(2^{\prime}\right)$. Moreover, we can write $\beta=\sum_{q \in M^{\top}} x_{q} \gamma_{q}$, where $x_{q}$ lies in $H^{*}(B T ; \mathbb{Q})$ for all $q$. If $\beta$ is integral, then each $x_{q}$ lies in $H^{*}(B T ; \mathbb{Z})$. 
If $x_{\mathrm{q}}=0$ for all $\mathrm{q} \in M^{\top}$ so that $\Psi(\mathrm{q})<\Psi(\mathrm{p})$, then properties (1) and $\left(2^{\prime}\right)$ together imply that $\beta(p)=x_{p} \Lambda_{p}^{-}$. Otherwise, there exists $q \in M^{\top}$ so that $\Psi(q)<\Psi(p)$ and $x_{q} \neq 0$, but $x_{r}=0$ for all $r$ such that $\Psi(r)<\Psi(q)$. Hence $\beta(q)=0$ and $\beta(q)=x_{q} \wedge_{q}^{-}$, which is impossible.

This corollary leads to the following properties of canonical classes.

Lemma 2.7. Let a torus $\mathrm{T}$ acts on a compact oriented manifold $\mathrm{M}$ with isolated fixed points, and let $\Psi: M \rightarrow \mathbb{R}$ be a formal moment map. For all $p \in M^{\top}$, the canonical class $\alpha_{p}$ is uniquely determined by the orientation on $v^{-}(p)$.

Proof. Fix an orientation on $v^{-}(p)$ and let $\alpha_{p}$ and $\alpha_{p}^{\prime}$ be canonical classes for $p \in M^{\top}$. Consider the class $\beta=\alpha_{p}-\alpha_{p}^{\prime} \in H_{T}^{2 \lambda(p)}(M ; \mathbb{Q})$. Assume $\beta \neq 0$. Then, since the restriction map from $\mathrm{H}_{\mathrm{T}}^{*}(M ; \mathbb{Z})$ to $\mathrm{H}_{\mathrm{T}}^{*}\left(M^{\top} ; \mathbb{Z}\right)$ is injective, there exists $q \in M^{\top}$ such that $\beta(q) \neq 0$ but $\beta(r)=0$ for all $r \in M^{\top}$ satisfying $\Psi(r)<\Psi(q)$. By the definition of canonical class, $\beta(s)=0$ for all $s \in M^{\top}$ such that $\lambda(s) \leq \lambda(p)$; therefore $\lambda(q)>\lambda(p)$. But this contradicts Corollary 2.6.

Lemma 2.8. Let a torus $\mathrm{T}$ act on a compact oriented manifold $\mathrm{M}$ with isolated fixed points, and let $\Psi: M \rightarrow \mathbb{R}$ be a formal moment map. If $\alpha_{\mathrm{p}} \in$ $\mathrm{H}_{\mathrm{T}}^{2 \lambda(p)}(M ; \mathbb{Q})$ is a canonical class for a fixed point $\mathrm{p}$, then $\alpha_{\mathrm{p}}$ also satisfies the property:

$\left(2^{\prime}\right) \alpha_{p}(q)=0$ for all $q \in M^{\top} \backslash\{p\}$ such that $\Psi(q) \leq \Psi(p)$.

Proof. There exists a point $q \in M^{\top}$ so that $\alpha_{p}(q) \neq 0$ but $\alpha_{p}(r)=0$ for all $r \in M^{\top}$ so that $\Psi(r)<\Psi(q)$. By the definition of canonical classes, the fact that $\alpha_{p}(q) \neq 0$ implies that either $q=p$ or $\lambda(q)>\lambda(p)$. In the latter case, Corollary 2.6 implies that $\alpha_{p}(q)=0$. Thus $q=p$.

This has the following important consequence.

Corollary 2.9. Let a torus $\mathrm{T}$ act on a compact oriented manifold $\mathrm{M}$ with isolated fixed points, and let $\Psi: M \rightarrow \mathbb{R}$ be a formal moment map. Define an oriented graph with vertex set $\mathrm{V}=\mathrm{M}^{\top}$ and edge set

$$
E=\left\{\left(r, r^{\prime}\right) \in M^{\top} \times M^{\top} \mid \lambda\left(r^{\prime}\right)-\lambda(r)=1 \text { and } \alpha_{r}\left(r^{\prime}\right) \neq 0\right\} .
$$

If there exists a path in ( $\mathrm{V}, \mathrm{E})$ from a point $\mathrm{p}$ to a different point $\mathrm{q}$, then $\Psi(p)<\Psi(q)$.

Finally, canonical classes do exist in a number of important special cases. For example, the following lemma is a special case of Lemma 1.13 in [MT].

Lemma 2.10 (McDuff-Tolman). Let the circle $\mathrm{S}^{1}$ act on a compact oriented manifold $M$ with isolated fixed points, and let $\Psi: M \rightarrow \mathbb{R}$ be a formal 
moment map. Then there exists a canonical class $\alpha_{p} \in \mathrm{H}_{\mathrm{S}^{1}}^{*}(\mathrm{M} ; \mathbb{Q})$ for all $p \in M^{\top}$.

Note that if $\Psi$ is a formal moment map then $-\Psi$ is also a formal moment map. If the index of $\Psi$ at $q \in M^{\top}$ is $2 \lambda(q)$, the index of $-\Psi$ at $q$ is $\operatorname{dim}(M)-$ $2 \lambda(q)$. Finally, the tangent bundle at $q$ is an oriented real representation of $\mathrm{T}$; let $\Lambda_{\mathrm{q}}$ denote the product of the weights of this representation. As one might expect, canonical classes for $\Psi$ exist for all points $p \in M^{\top}$ exactly when they exist for $-\Psi$ for all points.

Lemma 2.11. Let a torus $\mathrm{T}$ act on a compact oriented manifold $M$ with isolated fixed points and let $\Psi: M \rightarrow \mathbb{R}$ be a formal moment map. If there exists a canonical class $\alpha_{\mathrm{p}} \in \mathrm{H}_{\mathrm{T}}^{2 \lambda(p)}(M ; \mathbb{Q})$ with respect to $\Psi$ for each $\mathrm{p} \in \mathrm{M}^{\top}$, then there exists a canonical class $\beta_{\mathrm{q}} \in \mathrm{H}_{\mathrm{T}}^{\operatorname{dim}(M)-2 \lambda(\mathrm{q})}(\mathrm{M} ; \mathbb{Q})$ with respect to $-\Psi$ for each $\mathrm{q} \in \mathrm{M}^{\top}$. Moreover, $\beta_{\mathrm{q}}$ is integral for all $\mathrm{q} \in \mathrm{M}^{\top}$ if and only if $\alpha_{\mathrm{p}}$ is integral for all $\mathrm{p} \in \mathrm{M}^{\mathrm{\top}}$.

Proof. To begin, fix an orientation on $v^{+}(r)$ for all $r \in M^{\top}$, and consider the formal moment map $-\Psi$. We need to find a class $\beta_{\mathrm{q}} \in \mathrm{H}_{\mathrm{T}}^{\operatorname{dim}(M)-2 \lambda(\mathrm{q})}(M ; \mathbb{Z})$ such that

(1) $\beta_{\mathrm{q}}(\mathrm{q})=\Lambda_{\mathrm{q}}^{+}$and

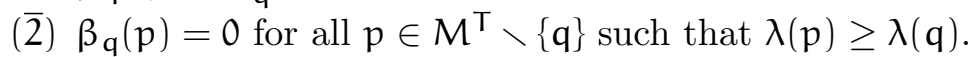

By Lemma 2.5, for all $r \in M^{\top}$ there exists a cohomology class $\gamma_{r} \in$ $\mathrm{H}_{\mathrm{T}}^{\operatorname{dim}(M)-2 \lambda(\mathrm{r})}(M ; \mathbb{Z})$ such that

(1) $\gamma_{\mathrm{r}}(\mathrm{r})=\Lambda_{\mathrm{r}}^{+}$and

$\left(\overline{2}^{\prime}\right) \gamma_{\mathrm{r}}(p)=0$ for all $p \in M^{\top} \backslash\{r\}$ so that $\Psi(p) \geq \Psi(r)$.

Now let $\beta_{\mathrm{q}}$ be any class which satisfies $(\overline{1})$ and $\left(\overline{2}^{\prime}\right)$ for q. Define

$$
\mathcal{L}:=\left\{p \in M^{\top} \mid \lambda(p) \geq \lambda(q) \text { but } \beta_{q}(p) \neq 0\right\} .
$$

If $\mathcal{L}=\emptyset$, we are done. Otherwise, let $p \in \mathcal{L}$ be an element which maximizes $\left.\Psi\right|_{\mathcal{L}}$. Then

$$
\left(\alpha_{p} \cup \beta_{q}\right)(p)=\Lambda_{p}^{-} \beta_{q}(p),
$$

where $\Lambda_{\mathrm{p}}^{-}$is the product of the weights on $v^{-}(p)$ with respect to the orientation compatible with $\alpha_{p}$. Now consider any fixed point $r \neq p$. If $\lambda(r) \leq \lambda(q)$, then $\lambda(r) \leq \lambda(p)$ and so $\alpha_{p}(r)=0$. Similarly, if $\Psi(r) \leq \Psi(p)$ then $\alpha_{p}(r)=0$ by Lemma 2.7. On the other hand, if $\lambda(r) \geq \lambda(q)$ and $\Psi(r)>\Psi(p)$ then $\beta_{\mathrm{q}}(\mathrm{r})=0$ since $\mathrm{p}$ maximizes $\left.\Psi\right|_{\mathcal{L}}$. Thus the product

$$
\left(\alpha_{p} \cup \beta_{q}\right)(r)=0 \text { for all } r \in M^{\top} \backslash\{p\} .
$$


We now integrate using the Atiyah-Bott-Berline-Vergne localization formula to obtain

$$
\int_{M} \alpha_{p} \cup \beta_{q}=\frac{\Lambda_{p}^{-} \beta_{q}(p)}{\Lambda_{p}}= \pm \frac{\beta_{q}(p)}{\Lambda_{p}^{+}} \in H^{*}(B T ; \mathbb{Q}) .
$$

This expression is integral if $\alpha_{\mathrm{p}}$ and $\beta_{\mathrm{q}}$ are both integral. Hence, the class $\beta_{\mathrm{q}}^{\prime}=\beta_{\mathrm{q}}-\left(\frac{\beta_{\mathrm{q}}(\mathrm{p})}{\Lambda_{\mathrm{p}}^{+}}\right) \beta_{\mathrm{p}}$ satisfies

- $\beta_{\mathrm{q}}^{\prime}(p)=0$,

- $\beta_{\mathrm{q}}^{\prime}(r)=0$ for all $r \in M^{\top} \backslash\{p\}$ such that $\Psi(r) \geq \Psi(p)$, and

- in particular, $\beta_{\mathrm{q}}^{\prime}$ also satisfies $(\overline{1})$ and $\left(\overline{2}^{\prime}\right)$ for $\mathrm{q}$.

The result now follows by induction.

Remark 2.12. Assume that for each $p \in M^{\top}$, there exists a canonical class $\alpha_{\mathfrak{p}} \in \mathrm{H}_{\mathrm{T}}^{2 \lambda(p)}(M)$ with respect to $\Psi$ which is compatible with a chosen orientation on $v^{-}(p)$. Let $\beta_{q} \in H_{T}^{\operatorname{dim}(M)-2 \lambda(q)}(M)$ be a canonical class with respect to $-\Psi$ for each $q \in M^{\top}$ described in Lemma 2.11. Clearly, $\beta_{\mathrm{q}}$ can be chosen to be compatible with the orientation of $v^{+}(q)$ induced by the orientations on $M$ and on $v^{-}(q)$. Then, since $\Lambda_{p}=\Lambda_{p}^{+} \Lambda_{p}^{-}$, the sets $\left\{\alpha_{p}\right\}$ and $\left\{\beta_{\mathrm{q}}\right\}$ are dual basis for $\mathrm{H}_{\mathrm{T}}^{*}(M)$ as an $\mathrm{H}_{\mathrm{T}}^{*}(M)$-module under the intersection pairing. To see this, note that if $\lambda(q)>\lambda(p)$, then $\int_{M} \alpha_{p} \beta_{q}=0$ by degree considerations. If $\lambda(q) \leq \lambda(p)$ and $p \neq q$, then $\left(\alpha_{p} \cup \beta_{q}\right)(r)=0$ for all $r \in$ $M^{\top}$ by the definition of canonical class, and so $\int_{M} \alpha_{p} \beta_{q}=0$ by the AtiyahBott-Berline-Vergne localization formula. Finally, by a similar argument, $\int_{M} \alpha_{p} \cup \beta_{p}=1$.

\section{Proof of Theorem 1.2}

We are now ready to prove Theorem 1.2. Let a torus $\mathrm{T}$ acts on a compact symplectic manifold $(M, \omega)$ with isolated fixed points and moment map $\Phi: M \rightarrow \mathfrak{t}^{*}$. Let $\Psi=\Phi^{\xi}$ be a generic component of the moment map. Assume that there exists a canonical class $\alpha_{p} \in H_{T}^{2 \lambda(p)}(M ; \mathbb{Q})$ for all $p \in M^{\top}$. Define an oriented graph with vertex set $V=M^{\top}$ and edge set

$$
E=\left\{\left(r, r^{\prime}\right) \in M^{\top} \times M^{\top} \mid \lambda\left(r^{\prime}\right)-\lambda(r)=1 \quad \text { and } \quad \alpha_{r}\left(r^{\prime}\right) \neq 0\right\} .
$$

We need to show that for all $p$ and $q$ in $M^{\top}$,

$$
\alpha_{p}(q)=\Lambda_{q}^{-} \sum_{r \in \Sigma_{p}^{q}} \prod_{i=1}^{|r|} \frac{\Phi\left(r_{i}\right)-\Phi\left(r_{i-1}\right)}{\Phi(q)-\Phi\left(r_{i-1}\right)} \frac{\alpha_{r_{i-1}}\left(r_{i}\right)}{\Lambda_{r_{i}}^{-}}
$$

where $\Sigma_{p}^{q}$ denotes the set of paths from $p$ to $q$ in $(V, E)$. By Lemma 2.11, for all $\mathrm{q} \in \mathrm{M}^{\top}$, there exists a class $\beta_{\mathrm{q}} \in \mathrm{H}_{\mathrm{T}}^{\operatorname{dim}(M)-2 \lambda(\mathrm{q})}(M ; \mathbb{Q})$ satisfying 


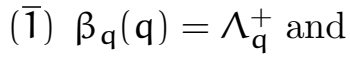

$(\overline{2}) \beta_{q}(p)=0$ for all $p \in M^{\top} \backslash\{q\}$ such that $\lambda(p) \geq \lambda(q)$.

In our proof of (3.2), we will also show that

$$
\beta_{q}(p)=\Lambda_{p}^{+} \sum_{r \in \Sigma_{p}^{q}} \prod_{i=1}^{|r|} \frac{\Phi\left(r_{i}\right)-\Phi\left(r_{i-1}\right)}{\Phi(p)-\Phi\left(r_{i}\right)} \frac{\alpha_{r_{i-1}}\left(r_{i}\right)}{\Lambda_{r_{i}}^{-}}
$$

for all fixed points $p$ and $q$.

Consider first the case that $\lambda(q)-\lambda(p) \leq 0$. If $p \neq q$, then $\alpha_{p}(q)=0$ by definition. Moreover, there are no paths from $p$ to $q$ in $(V, E)$, and so the right-hand side of (3.2) vanishes, as required. If $p=q$, then $\alpha_{p}(q)=$ $\alpha_{p}(p)=\Lambda_{p}^{-}$. In this case, the right-hand side of (3.2) is a sum over one degenerate path $\mathbf{r}=(p)$, and the product is the empty product, so the total contribution is $\Lambda_{\mathrm{p}}^{-}$. Thus (3.2) also holds in this case. A nearly identical argument proves that (3.3) is satisfied.

Next, suppose that $\lambda(q)-\lambda(p)=1$. If $\alpha_{p}(q) \neq 0$, then there is one path $(p, q)$ from $p$ to $q$, and the right-hand side of $(3.2)$ is $\alpha_{p}(q)$. On the other hand, if $\alpha_{p}(q)=0$, then $(p, q) \notin E$, and so the right-hand side of $(3.2)$ vanishes. To prove (3.3), note that by the definition of canonical class,

$$
\left(\alpha_{p} \cup \beta_{q}\right)(p)=\Lambda_{p}^{-} \beta_{q}(p) \text { and }\left(\alpha_{p} \cup \beta_{q}\right)(q)=\Lambda_{q}^{+} \alpha_{p}(q) \text {. }
$$

Now consider any fixed point $r$ which is not $p$ or $q$. If $\lambda(r) \leq \lambda(p)$ then $\alpha_{p}(r)=0$, while if $\lambda(r) \geq \lambda(q)$, then $\beta_{q}(r)=0$. Therefore,

$$
\left(\alpha_{p} \cup \beta_{q}\right)(r)=0 \quad \text { for all } r \in M^{\top} \backslash\{p, q\} \text {. }
$$

Since $\operatorname{deg}\left(\alpha_{p} \cup \beta_{q}\right)=\operatorname{dim}(M)-2<\operatorname{dim}(M)$, the integral of $\alpha_{p} \cup \beta_{q}$ over $M$ is zero. Thus, by the Atiyah-Bott-Berline-Vergne localization theorem,

$$
\int_{M} \alpha_{p} \cup \beta_{q}=\frac{\beta_{q}(p) \Lambda_{p}^{-}}{\Lambda_{p}}+\frac{\alpha_{p}(q) \Lambda_{q}^{+}}{\Lambda_{q}}=0 .
$$

Therefore,

$$
\beta_{\mathrm{q}}(\mathrm{p})=-\Lambda_{\mathrm{p}}^{+} \frac{\alpha_{\mathrm{p}}(\mathrm{q})}{\Lambda_{\mathrm{q}}^{-}}
$$

Fix $k>1$, and assume that (3.2) and (3.3) hold for all fixed points $p$ and $q$ so that $\lambda(q)-\lambda(p)<k$. Consider fixed points $p$ and $q$ so that $\lambda(q)-\lambda(p)=k$. We will prove that (3.2) and (3.3) follow for this $p$ and $q$.

Suppose first that $\Phi(p)=\Phi(q)$; a fortiori $\Psi(p)=\Psi(q)$. Then the lefthand sides of (3.2) and (3.3) vanish by Lemma 2.8. Since there is no path from $p$ to $q$ by Lemma 2.9 , the right-hand sides also vanish.

So assume instead that $\Phi(p) \neq \Phi(q)$. Let $\widetilde{\omega}=\omega+\Phi-\Phi(p)$ be an equivariant extension of $\omega$. Since $\widetilde{\omega}(r)=\Phi(r)-\Phi(p)$ for all $r \in M^{\top}$,

$$
\left(\alpha_{p} \cup \beta_{q} \cup \widetilde{\omega}\right)(q)=\Lambda_{q}^{+} \alpha_{p}(q)(\Phi(q)-\Phi(p)) \quad \text { and } \quad\left(\alpha_{p} \cup \beta_{q} \cup \widetilde{\omega}\right)(p)=0 \text {. }
$$


Since $k>1, \operatorname{deg}\left(\alpha_{p} \cup \beta_{q} \cup \widetilde{\omega}\right)=\operatorname{dim}(M)-2 k+2<\operatorname{dim}(M)$, and so the integral of $\alpha_{p} \cup \beta_{q} \cup \widetilde{\omega}$ over $M$ is zero. Therefore, by the Atiyah-BottBerline-Vergne localization formula,

$$
\int_{M} \alpha_{p} \cup \beta_{q} \cup \widetilde{\omega}=\frac{\Phi(q)-\Phi(p)}{\Lambda_{q}^{-}} \alpha_{p}(q)+\sum_{r \neq p, q} \frac{\left(\alpha_{p} \cup \beta_{q} \cup \widetilde{\omega}\right)(r)}{\Lambda_{r}}=0 .
$$

Since $\Phi(p) \neq \Phi(q)$, we can solve the above equation for $\alpha_{p}(q)$;

$$
\alpha_{p}(q)=\frac{-\Lambda_{q}^{-}}{\Phi(q)-\Phi(p)} \sum_{r \neq p, q} \frac{\left(\alpha_{p} \cup \beta_{q} \cup \widetilde{\omega}\right)(r)}{\Lambda_{r}} .
$$

Consider any fixed point $r \neq p$ or $q$. Assume first that $\lambda(p)<\lambda(r)<\lambda(q)$, and let $l=\lambda(r)-\lambda(p)$. By the inductive assumption

$$
\alpha_{p}(r)=\Lambda_{r}^{-} \sum_{\left(s_{0}, \ldots, s_{l}\right)} \in \sum_{p}^{r} \prod_{i=1}^{l} \frac{\Phi\left(s_{i}\right)-\Phi\left(s_{i-1}\right)}{\Phi(r)-\Phi\left(s_{i-1}\right)} \frac{\alpha_{s_{i-1}}\left(s_{i}\right)}{\Lambda_{s_{i}}^{-}}
$$

and

$$
\beta_{\mathrm{q}}(\mathrm{r})=\Lambda_{\mathrm{r}}^{+} \sum_{\left(s_{1}, \ldots, s_{k}\right) \in \Sigma_{r}^{\mathrm{q}}} \prod_{i=l+1}^{k} \frac{\Phi\left(s_{i}\right)-\Phi\left(s_{i-1}\right)}{\Phi(r)-\Phi\left(s_{i}\right)} \frac{\alpha_{s_{i-1}}\left(s_{i}\right)}{\Lambda_{s_{i}}^{-}}
$$

Therefore, if $\Sigma_{p}^{q}(r)$ denotes the set of paths in $E$ from $p$ to $q$ that pass through $r$, then

$$
\begin{aligned}
\left(\alpha_{p} \cup \beta_{q} \cup \widetilde{\omega}\right)(r)= & \Lambda_{r}^{+} \Lambda_{r}^{-}(\Phi(r)-\Phi(p)) \\
& \times \sum_{s \in \Sigma_{p}^{q}(r)} \frac{\prod_{i=1}^{k}\left[\Phi\left(s_{i}\right)-\Phi\left(s_{i-1}\right)\right] \frac{\alpha_{s_{i-1}}\left(s_{i}\right)}{\Lambda_{s_{i}}^{-}}}{\prod_{i \in\left\{0, \ldots, k_{j} \backslash\{l\}\right.} \Phi(r)-\Phi\left(s_{i}\right)} \\
= & \Lambda_{r} \sum_{\mathbf{s} \in \Sigma_{p}^{q}(r)} \prod_{i=1}^{k}\left[\Phi\left(s_{i}\right)-\Phi\left(s_{i-1}\right)\right] \frac{\alpha_{s_{i-1}}\left(s_{i}\right)}{\Lambda_{s_{i}}^{-}} \\
& \times \prod_{i \neq l} \frac{1}{\Phi(r)-\Phi\left(s_{i}\right)},
\end{aligned}
$$

where we use the expression $\prod_{i \neq l}$ as shorthand for $\prod_{i \in\left\{1, \ldots, k_{j} \backslash\{l\}\right.}$. On the other hand, if $\lambda(r) \leq \lambda(p)$ or $\lambda(q) \leq \lambda(p)$, then $\left(\alpha_{p} \cup \beta_{q} \cup \widetilde{\omega}\right)(r)=0$. By Lemma 2.9, the right-hand side of (3.5) also vanishes. Therefore, (3.5) holds for all $r \in M^{\top} \backslash\{p, q\}$. 
Substituting (3.5) into (3.4), we see that

$$
\begin{aligned}
\alpha_{p}(\mathbf{q})= & \frac{-\Lambda_{\mathbf{q}}^{-}}{\Phi(\mathbf{q})-\Phi(p)} \sum_{r \in M^{\top} \backslash\{p, q\}} \\
& \times\left(\sum_{\mathbf{s} \in \Sigma_{p}^{q}(r)} \prod_{i=1}^{k}\left[\Phi\left(s_{i}\right)-\Phi\left(s_{i-1}\right)\right] \frac{\alpha_{s_{i-1}}\left(s_{i}\right)}{\Lambda_{s_{i}}^{-}} \prod_{i \neq l} \frac{1}{\Phi(r)-\Phi\left(s_{i}\right)}\right) \\
= & \frac{-\Lambda_{\mathbf{q}}^{-}}{\Phi(\mathbf{q})-\Phi(p)} \sum_{\mathbf{s} \in \Sigma_{p}^{q}} \prod_{i=1}^{k}\left[\Phi\left(s_{i}\right)-\Phi\left(s_{i-1}\right)\right] \frac{\alpha_{s_{i-1}}\left(s_{i}\right)}{\Lambda_{s_{i}}^{-}} \\
& \times\left(\sum_{l=1}^{k-1} \prod_{i \neq l} \frac{1}{\Phi\left(s_{l}\right)-\Phi\left(s_{i}\right)}\right) \\
= & \frac{-\Lambda_{\mathbf{q}}^{-}}{\Phi(\mathbf{q})-\Phi(p)} \sum_{\mathbf{s} \in \Sigma_{p}^{q}} \prod_{i=1}^{k}\left[\Phi\left(s_{i}\right)-\Phi\left(s_{i-1}\right)\right] \frac{\alpha_{s_{i-1}}\left(s_{i}\right)}{\Lambda_{s_{i}}^{-}} \\
& \times\left(-\prod_{i=1}^{k-1} \frac{1}{\Phi\left(s_{k}\right)-\Phi\left(s_{i}\right)}\right) \\
= & \Lambda_{q}^{-} \sum_{\mathbf{s} \in \Sigma_{p}^{q}}^{k} \prod_{i=1}^{k}\left[\Phi\left(s_{i}\right)-\Phi\left(s_{i-1}\right)\right] \frac{\alpha_{s_{i-1}}\left(s_{i}\right)}{\Lambda_{s_{i}}^{-}} \prod_{i=1}^{k} \frac{1}{\Phi(q)-\Phi\left(s_{i-1}\right)}
\end{aligned}
$$

where the third equality is by Lemma 3.3. The proof of (3.3) is nearly identical.

Remark 3.1. In fact, the same proof works if a torus $T$ acts on a manifold $M$ with isolated fixed points, $\Psi: M \rightarrow \mathbb{R}$ is a formal moment map, and $\widetilde{\eta}=\eta+\Phi$ is any closed equivariant 2 -form (not necessarily symplectic) so that $\Phi(p) \neq \Phi(q)$ for every pair of fixed points $p$ and $q$ so that there is a path (of length two of more) in (V,E) from $p$ to $q$.

The following corollary is immediate.

Corollary 3.2. Consider the situation described in Theorem 1.2. If $\Psi=\Phi^{\xi}$ achieves its minimum value at $\mathrm{p}$, then for any fixed point $\mathrm{q}$,

$$
1=\Lambda_{\mathrm{q}}^{-} \sum_{\mathbf{r} \in \Sigma_{p}^{\mathrm{q}}} \prod_{i=1}^{|\mathbf{r}|} \frac{\Phi\left(\mathrm{r}_{\mathrm{i}}\right)-\Phi\left(\mathrm{r}_{\mathrm{i}-1}\right)}{\Phi(\mathrm{q})-\Phi\left(\mathrm{r}_{\mathrm{i}-1}\right)} \frac{\alpha_{\mathrm{r}_{i-1}}\left(\mathrm{r}_{\mathrm{i}}\right)}{\Lambda_{\mathrm{r}_{\mathrm{i}}}^{-}}
$$

where $\Sigma_{\mathrm{p}}^{\mathrm{q}}$ denotes the paths in $(\mathrm{V}, \mathrm{E})$ from $\mathrm{p}$ to $\mathrm{q}$. In particular, every fixed point is connected by a path in ( $\mathrm{V}, \mathrm{E})$ to the minimum (and to the maximum). 
Proof. Since $\lambda(p)=0, \alpha_{p}=1$; hence, $\alpha_{p}(q)=1$ for all fixed points $q$.

Our proof of Theorem 1.2 relies on the following fact, which was also proved in $[\mathbf{G Z}]$ using different techniques.

Lemma 3.3. Given $\mathrm{k}>1$ distinct vectors $v_{1}, \ldots, v_{\mathrm{k}}$ in a vector space $\mathrm{V}$,

$$
\sum_{l=1}^{k} \prod_{i \neq l} \frac{1}{v_{i}-v_{l}}=0 .
$$

Proof. The $\left(\mathrm{S}^{1}\right)^{\mathrm{k}}$ action on $\mathbb{C}^{\mathrm{k}}$ induces a symplectic action of on $\mathbb{C P}^{\mathrm{k}-1}$ with fixed points $p_{1}, \ldots, p_{k}$. so that the weights at $p_{l}$ are $\left\{x_{i}-x_{l}\right\}_{i \neq l} \in$ $H^{*}\left(\left(B\left(S^{1}\right)^{k} ; \mathbb{Z}\right)=\mathbb{Z}\left[x_{1}, \ldots, x_{k}\right]\right.$. Since $\operatorname{deg}(1)=0<2 k-2$, the integral of 1 over $\mathbb{C P}^{k-1}$ is 0 . Therefore, by the Atiyah-Bott-Berline-Vergne localization theorem, the following equation holds in the field of rational functions $\mathbb{Q}\left(x_{1}, \ldots, x_{k}\right)$ :

$$
\int_{\mathbb{C P}^{k-1}} 1=\sum_{l=1}^{k} \prod_{i \neq l} \frac{1}{x_{i}-x_{l}}=0 .
$$

Since the $v_{i}$ 's are distinct, the claim follows easily.

\section{The GKM case}

The main goal of this section is to prove Theorem 1.6. In fact, this theorem is an immediate consequence of Theorem 1.2 and the theorem below.

Theorem 4.1. Let $(\mathrm{M}, \omega, \Phi)$ be GKM space, and let $\left(\mathrm{V}, \mathrm{E}_{\mathrm{GKM}}\right)$ be the associated GKM graph. Let $\Psi=\Phi^{\xi}$ be a generic component of the moment map; assume that $\Psi$ is index increasing. Then

- There exists a canonical class $\alpha_{\mathrm{p}} \in \mathrm{H}_{\mathrm{T}}^{2 \lambda(\mathrm{p})}(\mathrm{M} ; \mathbb{Z})$ for all $\mathrm{p} \in \mathrm{M}^{\mathrm{T}}$.

- Given $\mathrm{p}$ and $\mathrm{q}$ in $\mathrm{M}^{\top}$ such that $\lambda(\mathrm{q})-\lambda(\mathrm{p})=1$,

$$
\alpha_{p}(q)= \begin{cases}\Lambda_{q}^{-\frac{\Theta(p, q)}{\eta(p, q)}} & \text { if }(p, q) \in E_{G K M}, \\ 0 & \text { if }(p, q) \notin E_{G K M} .\end{cases}
$$

- $\Theta\left(r, r^{\prime}\right) \in \mathbb{Z} \backslash\{0\}$ for all $\left(r, r^{\prime}\right) \in E_{G K M}$.

Remark 4.2. Conversely, if there exists a canonical class $\alpha_{p} \in H_{T}^{*}(M ; \mathbb{Q})$ for all $p \in M^{\top}$, then $\Psi$ is index increasing. To see this, suppose that there exists an edge $(p, q) \in E_{G K M}$ so that $\Psi(p)<\Psi(q)$ and $\lambda(p) \geq \lambda(q)$. On the one hand, since $\alpha_{p}$ is a canonical class this implies that $\alpha_{p}(q)=0$. On the other hand, compatibility along $(p, q)$ guarantees that $\alpha_{p}(q)-\alpha_{p}(p)$ is a multiple of $\eta(p, q)$. Sine $\alpha_{p}(p)=\Lambda_{p}^{-}$is not a multiple of $\eta(p, q)$, this implies that $\alpha_{\mathbf{p}}(\mathbf{q}) \neq 0$. 
Remark 4.3. There are several situations where we can immediately conclude that $\Psi$ is index increasing.

For example, if there is an T-invariant Palais-Smale metric $g$ on $M$, then $\Psi$ is index increasing. To see this, consider an edge $(p, q) \in E_{G K M}$ such that $\Psi(p)<\Psi(q)$. There exists a codimension one subgroup $K \subset T$ so that $p$ and $q$ are contained in the same component $N$ of $M^{K}$. Since the metric is T-invariant, $N \backslash\{p, q\}$ must be contained in both the flow up from $p$ and the flow down from q. Since these flows intersect transversally, this implies that the intersection has dimension $2 \lambda(q)-2 \lambda(p) \geq 2$, which proves $\lambda(p)<\lambda(q)$.

Similarly, if $H^{2 i}(M ; \mathbb{Q})=\mathbb{Q}$ for all $i$ such that $0 \leq 2 i \leq \operatorname{dim}(M)$, then Proposition 3.4 in [To2] implies that every generic component of the moment map is index increasing.

Our proof of the theorem above relies heavily on a technical proposition, Proposition 4.4, for which we need a few definitions. Let $(M, \omega, \Phi)$ be a GKM space, and let $\left(V, E_{G K M}\right)$ be the associated GKM graph. Fix a generic $\xi \in \mathfrak{t}$ and consider the Morse function $\Psi=\Phi^{\xi}$.

A path $r$ in $\left(V, E_{G K M}\right)$ is ascending if $\Psi\left(r_{i}\right) \leq \Psi\left(r_{i+1}\right)$ for all $i$ such that $0 \leq i \leq|r|$; it is descending if $\Psi\left(r_{i}\right) \geq \Psi\left(r_{i+1}\right)$ for all such $i$. Given $p \in M^{\top}$, the stable set of $p$, denoted $V_{p}$, is the set of $q \in V$ such that there exists an ascending path from $p$ to $q$ in $\left(V, E_{G K M}\right)$; the unstable set of $p$, denoted $V^{p}$, is the set of $q \in V$ such that there exists an descending path from $p$ to q. Note that $p$ itself lies in both $V_{p}$ and $V^{p}$. Moreover, since $\left(r, r^{\prime}\right) \in E_{G K M}$ exactly if $\left(r^{\prime}, r\right) \in E_{G K M}, p \in V_{q}$ exactly if $q \in V^{p}$.

Proposition 4.4. Let $(M, \omega, \Phi)$ be a GKM space, and let $\Psi=\Phi^{\xi}$ be a generic component of the moment map.

(a) For every fixed point $\mathrm{p}$, there exists a class $\alpha_{\mathrm{p}} \in \mathrm{H}_{\mathrm{T}}^{2 \lambda(\mathrm{p})}(\mathrm{M} ; \mathbb{Z})$ such that

(1) $\alpha_{p}(p)=\Lambda_{p}^{-}$and

$\left(2^{\prime \prime}\right) \alpha_{p}(q)=0$ for all $q \in M^{\top} \backslash V_{p}$.

(b) Given a class $\beta \in \mathrm{H}_{\mathrm{T}}^{*}(\mathrm{M} ; \mathbb{Z})$ and point $\mathrm{q} \in \mathrm{M}^{\top}$,

$$
\beta(q)=x \prod_{\substack{\left.(r, q) \in E_{G K M} \\ \beta\right|_{V}=0}} \eta(r, q), \quad \text { where } x \in H^{*}(B T ; \mathbb{Z}) .
$$

Proposition 4.4(a) is proved for rational classes in the more general setting of GKM graphs in $[\mathbf{G Z}]$.

Remark 4.5. Part (a) of this proposition is exactly what geometric intuition leads you to expect. To see this, fix a generic T-invariant metric on $M$. As the name suggests, the stable set $V_{p}$ should be the set of vertices which are in the closure of the stable manifold of $p$. Moreover, one should be able to adapt the Morse theoretic proof of Lemma 2.5 to directly prove 
that the class $\alpha_{\mathfrak{p}}$ which you construct is supported on the set of vertices in the closure of the stable manifold of $p$.

Remark 4.6. Part (b) is slightly more subtle. Given a class $\beta \in \mathrm{H}_{\mathrm{T}}^{*}(M ; \mathbb{Q})$ and an edge $\left(r, r^{\prime}\right) \in E_{G K M}, \beta(r)-\beta\left(r^{\prime}\right)$ is a rational multiple ${ }^{5}$ of $\eta\left(r, r^{\prime}\right)$. Since the weights at each fixed point are pairwise linearly independent, this immediately implies that for any fixed point $q$,

$$
\beta(q)=x \prod_{\substack{(r, q) \in E_{G K M} \\ \beta(r)=0}} \eta(r, q), \quad \text { where } x \in H^{*}(B T ; \mathbb{Q}) .
$$

However, the same statement is not true integrally. Although $\beta(q)$ must be an integral multiple of $\eta(r, q)$ for each $(r . q) \in E_{G K M}$ such that $\eta(r)=0$, it might not be an integral multiple of the product of these weights because the weights might not be pairwise relatively prime; see Example 4.7. Notice that expression (4.2) has fewer terms in the product.

Example 4.7. Let $S^{1} \times S^{1}$ act on $\mathbb{C P}^{1} \times \mathbb{C P}^{1}$ by

$$
\left(\mathrm{t}_{1}, \mathrm{t}_{2}\right) \cdot\left(\left[z_{0}: z_{1}\right],\left[w_{0}: w_{1}\right]\right)=\left(\left[\mathrm{t}_{1}^{2} z_{0}: z_{1}\right],\left[\mathrm{t}_{2}^{2} w_{0}: w_{1}\right]\right)
$$

let $\Phi: M \rightarrow \mathfrak{t}^{*}$ be the moment map. Let $\xi=(1,1) \in \mathbb{R}^{2}$ and let $\Psi=\Phi^{\xi}$. The associated GKM graph has four vertices,

$$
\begin{aligned}
& S S=([1: 0],[1: 0]), \quad S N=([1: 0],[0: 1]), \quad N S=([0: 1],[1: 0]), \\
& \quad \text { and } N N=([0: 1],[0: 1]) .
\end{aligned}
$$

There are four ascending edges, (SS, NS), (SN, NN), (SS, SN), and (NS, NN) (and hence also four descending edges). The first two have weight $2 x_{1}$, the latter two have weight $2 x_{2}$. There exists a class $\beta \in H_{T}^{4}(M ; \mathbb{Z})$ so that

$$
\beta(\mathrm{SN})=\beta(\mathrm{NS})=0 \text { and } \beta(\mathrm{SS})=\beta(\mathrm{NN})=2 \mathrm{x}_{1} x_{2} \text {. }
$$

Although $\beta(S N)=0,\left.\beta\right|_{V^{S N}} \neq 0$ because $V^{S N}=\{S N, S S\}$. Similarly, $\left.\beta\right|_{V S N} \neq 0$. Therefore, even though $\beta_{N N}=2 x_{1} x_{2}$ is not a multiple of $\Lambda_{\mathrm{NN}}^{-}=\eta(\mathrm{SN}, \mathrm{NN}) \eta(\mathrm{NS}, \mathrm{NN})=4 x_{1} x_{2}$, this example does satisfy (4.2) in Proposition 4.4.

We begin by showing that Theorem 4.1 follows from Proposition 4.4.

Proof of Theorem 4.1. Fix $p \in M^{\top}$. By Proposition 4.4, there exists a class $\alpha_{p} \in H_{T}^{2 \lambda(p)}(M ; \mathbb{Z})$ which satisfies properties (1) and $\left(2^{\prime \prime}\right)$. Since $\Psi$ is index increasing, $\lambda(q)>\lambda(p)$ for all $q \in V_{p} \backslash\{p\}$. Hence, $\alpha_{p}$ is a canonical class.

Consider $q \in M^{\top}$ such that $\lambda(q)=\lambda(p)+1$. If $(p, q) \notin E_{G K M}$, then $q \notin V_{p}$, and so $\alpha_{p}(q)=0$. Now assume that $(p, q) \in E_{G K M}$. There are

\footnotetext{
${ }^{5}$ In fact, a rational class $\beta \in \mathrm{H}_{\mathrm{T}}^{*}\left(M^{\top} ; \mathbb{Q}\right)$ is in the image of the restriction map $\iota^{*}$ : $\mathrm{H}_{\mathrm{T}}^{*}(M ; \mathbb{Q}) \rightarrow \mathrm{H}_{\mathrm{T}}^{*}\left(M^{\top} ; \mathbb{Q}\right)$ exactly if $\beta(q)-\beta(p)$ is a multiple of $\eta(p, q)$ for every edge $(p, q) \in E_{G K M}($ see $[\mathbf{G K M}])$.
} 
$\lambda(p)=\lambda(q)-1$ other edges $e_{1}, \ldots, e_{\lambda(p)} \in E_{G K M}$ of the form $e_{i}=\left(p_{i}, q\right)$ with $\Psi\left(p_{i}\right)<\Psi(q)$. Since $\Psi$ is index increasing, $\lambda\left(p_{i}\right) \leq \lambda(p)$ and so $V_{p} \cap V^{p_{i}}=\emptyset$ for all $i$. Thus $\alpha_{p} \mid v^{p_{i}}=0$ for all $i$. By Proposition $4.4, \alpha_{p}(q)$ is an integral multiple of $\prod_{i} \eta\left(p_{i}, q\right)=\frac{\Lambda_{q}^{-}}{\eta(p, q)}$; by degree considerations this implies that

$$
\alpha_{p}(q)=\theta \frac{\Lambda_{q}^{-}}{\eta(p, q)}, \quad \text { where } \theta \in \mathbb{Z} .
$$

On the other hand, compatibility along $(p, q)$ guarantees that $\alpha_{p}(q)-$ $\alpha_{p}(p)=\theta \Lambda_{q}^{-} / \eta(p, q)-\Lambda_{p}^{-}$is a multiple of $\eta(p, q)$, and hence $\theta$ satisfies

$$
\frac{\Lambda_{q}^{-}}{\eta(p, q)} \theta=\Lambda_{p}^{-} \bmod \eta(p, q) \text {. }
$$

Since $\Lambda_{p}^{-}$is not a multiple of $\eta(p, q)$, the integer $\theta$ is non-zero. Finally, a straightforward calculation shows that

$$
\theta=\frac{\rho_{\eta\left(r, r^{\prime}\right)}\left(\Lambda_{r}^{-}\right)}{\rho_{\eta\left(r, r^{\prime}\right)}\left(\frac{\Lambda_{r^{\prime}}^{-}}{\eta\left(r, r^{\prime}\right)}\right)}
$$

Hence, $\theta=\Theta\left(r, r^{\prime}\right)$.

We now turn to the proof of Proposition 4.4. We begin by establishing some terminology, after which we prove two key lemmas. Finally, we use these lemmas and an inductive argument on the dimension of $M$ to complete the proof.

Definition 4.8. Let $(M, \omega, \Phi)$ be a GKM space, and let $\Psi=\Phi^{\xi}$ be a generic component of the moment map. We say that $\beta \in \mathrm{H}_{\mathrm{T}}^{*}(M ; \mathbb{Z})$ is robustly zero at $r \in M^{\top}$ if $\left.\beta\right|_{\mathrm{V}^{r}}=0$. We say that $\beta$ is robustly integral at $\mathrm{q} \in \mathrm{M}^{\top}$ if

$$
\beta(q)=x \prod_{\substack{\left.(r, q) \in E_{G K M} \\ \beta\right|_{V^{r}=0}}} \eta(r, q), \quad \text { where } x \in H^{*}(B T ; \mathbb{Z}) .
$$

Thus Proposition 4.4(b) is the statement that every class $\beta \in \mathrm{H}_{\mathrm{T}}^{*}(M ; \mathbb{Z})$ is robustly integral at every point $\mathrm{q} \in \mathrm{M}^{\top}$. We first show that Proposition 4.4(b) implies 4.4(a).

Lemma 4.9. Let $(M, \omega, \Phi)$ be a GKM space, and let $\Psi=\Phi^{\xi}$ be a generic component of the moment map. Assume that every class $\beta \in \mathrm{H}_{\mathrm{T}}^{*}(\mathrm{M} ; \mathbb{Z})$ is robustly integral at every $\mathrm{q} \in \mathrm{M}^{\top}$. Then, for every fixed point $\mathrm{p}$, there exists a class $\alpha_{\mathrm{p}} \in \mathrm{H}_{\mathrm{T}}^{2 \lambda(\mathrm{p})}(\mathrm{M} ; \mathbb{Z})$ such that

(1) $\alpha_{p}(p)=\Lambda_{p}^{-}$and

$\left(2^{\prime \prime}\right) \alpha_{p}(q)=0$ for all $q \in M^{\top} \backslash v_{p}$. 
Proof. By Lemma 2.5, for all $\mathrm{q} \in \mathrm{M}^{\top}$ there exists a class $\gamma_{\mathrm{q}} \in \mathrm{H}_{\mathrm{T}}^{2 \lambda(\mathrm{q})}(\mathrm{M} ; \mathbb{Z})$

(1) $\gamma_{\mathrm{q}}(\mathrm{q})=\Lambda_{\mathrm{q}}^{-}$.

$\left(2^{\prime}\right) \gamma_{\mathbf{q}}(r)=0$ for all $r \in M^{\top} \backslash\{\mathbf{q}\}$ such that $\Psi(r) \leq \Psi(\mathbf{q})$.

Let $\alpha_{p} \in H_{T}^{2 \lambda(p)}(M ; \mathbb{Z})$ be any class which satisfies (1) and $\left(2^{\prime}\right)$ for $p$. Define

$$
\mathcal{L}=\left\{q \in M^{\top} \backslash v_{p} \mid \alpha_{p}(q) \neq 0\right\}
$$

If $\mathcal{L}=\emptyset$, we are done. Otherwise, let $q \in \mathcal{L}$ be an element which minimizes $\left.\Psi\right|_{\mathcal{L}}$. Consider any $r \in V^{q} \backslash\{\mathbf{q}\}$. Since $\Psi(r)<\Psi(\mathbf{q})$ and $\mathbf{q}$ is minimal, $r \notin \mathcal{L}$. Moreover, since $q \in V_{r}$ but $q \notin V_{p}$, we conclude that $r \in M^{\top} \backslash V_{p}$; hence $\alpha_{p}(r)=0$. Therefore, $\left.\alpha_{p}\right|_{V^{r}}=0$ for each edge $(r, q) \in E_{G K M}$ such that $\Psi(r)<\Psi(q)$. Since $\alpha_{p}$ is robustly integral at $q$ this implies that there exists $x \in H^{*}(B T ; \mathbb{Z})$ so that $\alpha_{p}(q)=x \Lambda_{q}^{-}=x \gamma_{q}(q)$. The difference $\alpha_{p}^{\prime}=\alpha_{p}-x \gamma_{q}$ satisfies

- $\alpha_{p}^{\prime}(q)=0$,

- $\alpha_{p}^{\prime}(r)=\alpha_{p}(r)$ for all $r \in M^{\top} \backslash\{\mathbf{q}\}$ such that $\Psi(r) \leq \Psi(\mathbf{q})$, and

- in particular, $\alpha_{p}^{\prime}$ also satisfies (1) and $\left(2^{\prime}\right)$ for $p$.

The result now follows by induction.

Now we show that Proposition 4.4(a) plus the assumption that all the $\alpha_{p}$ are robustly integral at all fixed points implies Proposition 4.4(b).

Lemma 4.10. Let $(M, \omega, \Phi)$ be a GKM space, and let $\Psi=\Phi^{\xi}$ be a generic component of the moment map. Assume that, for every fixed point $\mathrm{p}$, there exists a class $\alpha_{\mathrm{p}} \in \mathrm{H}_{\mathrm{T}}^{2 \lambda(\mathrm{p})}(\mathrm{M} ; \mathbb{Z})$ such that

(1) $\alpha_{p}(p)=\Lambda_{p}^{-}$,

$\left(2^{\prime \prime}\right) \alpha_{\mathrm{p}}(\mathrm{q})=0$ for all $\mathrm{q} \in \mathrm{M}^{\top} \backslash \mathrm{V}_{\mathrm{p}}$, and

(3) $\alpha_{p}$ is robustly integral at every point $q \in M^{\top}$.

Then every class $\beta \in \mathrm{H}_{\mathrm{T}}^{*}(M ; \mathbb{Z})$ is robustly integral at every point $\mathrm{q} \in \mathrm{M}^{\top}$.

Proof. Fix a class $\beta \in \mathrm{H}_{\mathrm{T}}^{*}(M ; \mathbb{Z})$. By Lemma 2.5 , we can write

$$
\beta=\sum_{p \in M^{\top}} x_{p} \alpha_{p}, \quad \text { where } x_{p} \in H^{*}(B T ; \mathbb{Z}) \forall p \in M^{\top} .
$$

Fix $r \in M^{\top}$ such that $\left.\beta\right|_{V^{r}}=0$. We claim that $x_{p}=0$ for all $p \in V^{r}$. If not, then there exists $p \in V^{r}$ so that $x_{p} \neq 0$ but $x_{q}=0$ for all $q \in V^{r}$ such that $\Psi(q)<\Psi(p)$. But then $\beta(q)=x_{q} \wedge_{q}^{-} \neq 0$, which contradicts the assumption that $\left.\beta\right|_{V^{r}}=0$. Therefore, if $x_{p} \neq 0$, then $p \notin V^{r}$, and so $V_{p} \cap V^{r}=\emptyset$; hence $\left.\alpha_{p}\right|_{V^{r}}=0$. Since each $\alpha_{p}$ is robustly integral at every point $\mathrm{q} \in \mathrm{M}^{\top}$, this completes the proof.

Proof of Proposition 4.4. Assume that Proposition 4.4 is true for all manifolds of dimension less than $\operatorname{dim} M$; we will prove that it is true for $M$. The result will then follow by induction. 
We first consider the case when $T$ acts on $M$ effectively. Choose any $\beta \in \mathrm{H}_{\mathrm{T}}^{*}(M ; \mathbb{Z})$ and fixed point $\mathrm{q} \in \mathrm{M}^{\top}$. By Lemma 4.9 , it is enough to prove that $\beta$ is robustly integral at $q$.

The proposition is obvious when $\operatorname{dim} M=2$. Hence, $\beta(q)$ is an integer multiple of $\eta(r, q)$ for every $(r, q) \in E_{G K M}$ with $\left.\beta\right|_{V^{r}}=0$. Since these weights are pairwise linearly independent, this implies that $\beta(q)$ is a rational multiple of

$$
\prod_{\substack{\left.(r, q) \in E_{G K M} \\ \beta\right|_{V^{r}=0}}} \eta(r, q) .
$$

Now consider any prime $k$ and natural number $l$ such that $k^{l}$ divides product (4.5). Let $\Gamma=\left\{\mathrm{t} \in \mathrm{T} \mid \mathrm{t}^{\mathrm{k}}=1\right\}$, and let $\mathrm{N} \subset \mathrm{M}$ be the component of $M^{\Gamma}$ containing $q$. Then $\left(N,\left.\omega\right|_{N},\left.\Phi\right|_{N}\right)$ is also a GKM space, and $\left.\Psi\right|_{N}=\left.\Phi^{\xi}\right|_{N}$ is a generic component of the moment map. Let $\left(V_{N},\left(E_{G K M}\right)_{N}\right)$ be the associated GKM graph. Given any edge $(r, q) \in E_{G K M}$, the weight $\eta(r, q)$ is a multiple of $k$ exactly if $(r, q) \in\left(E_{G K M}\right)_{N}$. Therefore, since $k^{l}$ divides product (4.5), it divides the (smaller) product

$$
\prod_{\substack{\left.(r, q) \in\left(E_{G K M}\right)_{N} \\ \beta\right|_{V}=0}} \eta(r, q) .
$$

Since $\left(E_{G K M}\right)_{N} \subset E_{G K M}$, if $\beta \in H_{T}^{*}(M ; \mathbb{Z})$ is robustly zero at $r \in V_{N}$, then $\iota_{N}^{*} \beta \in H_{T}^{*}(N ; \mathbb{Z})$ is robustly zero at $r$, where $\iota_{N}^{*}: H_{T}^{*}(M) \rightarrow H_{T}^{*}(N)$ is the restriction map. Moreover, since the $T$ action on $M$ is effective, $\operatorname{dim} N<\operatorname{dim} M$. By the inductive hypothesis, this implies that $\iota_{N}^{*} \beta(q)=$ $\beta(q)$ is an integral multiple of product (4.6). Therefore, since $k^{l}$ divides this product, $k^{l}$ also divides $\beta(\mathbf{q})$. This proves the proposition when $T$ acts effectively.

We now consider the general case. By Lemma 4.10, it is enough to show that for each $p$ there exists a class $\alpha_{p}$ which satisfies (1) and $\left(2^{\prime \prime}\right)$ and is robustly integral at every point $q \in M^{\top}$.

Let

$$
\operatorname{Stab}(M)=\{t \in T \mid t m=m \text { for all } m \in M\}
$$

and $\mathrm{T}^{\prime}=\mathrm{T} / \operatorname{Stab}(\mathrm{M})$. Let $\Pi: \mathrm{T} \rightarrow \mathrm{T}^{\prime}$ be the natural projection, and let $\pi: \mathfrak{t} \rightarrow \mathfrak{t}^{\prime}$ be the induced map on the lie algebras. Notice that $\pi$ takes the lattice $\ell \subset \mathfrak{t}$ to a sublattice of $\ell^{\prime} \subset \mathfrak{t}^{\prime}$. 
Since $T^{\prime}$ acts naturally on $M$, the map $\Pi: T \rightarrow T^{\prime}$ induces maps in equivariant cohomology ${ }^{6}$

$$
\Pi_{M}^{*}: \mathrm{H}_{\mathrm{T}^{\prime}}^{*}(M ; \mathbb{Z}) \longrightarrow \mathrm{H}_{\mathrm{T}}^{*}(M ; \mathbb{Z})
$$

and

$$
\Pi_{\mathrm{p}}^{*}: \mathrm{H}_{\mathrm{T}^{\prime}}^{*}(\{\mathrm{p}\} ; \mathbb{Z}) \longrightarrow \mathrm{H}_{\mathrm{T}}^{*}(\{\mathrm{p}\} ; \mathbb{Z}) \forall \mathrm{p} \in \mathrm{M}^{\top} .
$$

Since $M^{\top}=M^{T^{\prime}}$, these fit together into the following commutative diagram.

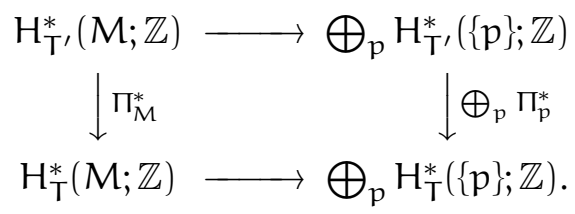

Moreover, for each $p \in M^{\top}$, in degree two the map $\Pi_{p}^{*}$ is identified with the dual map $\pi^{*}:\left(\mathfrak{t}^{\prime}\right)^{*} \rightarrow \mathfrak{t}^{*}$ under the natural identification of $\mathrm{H}_{\mathrm{T}}^{2}(\{p\} ; \mathbb{Z})$ and $\mathrm{H}_{\mathrm{T}^{\prime}}^{2}(\{\mathrm{p}\} ; \mathbb{Z})$ with the lattices $\ell^{*}$ and $\left(\ell^{\prime}\right)^{*}$, respectively.

Clearly, there exists a moment map $\Phi^{\prime}: M \rightarrow\left(\mathfrak{t}^{\prime}\right)^{*}$ so that $\left(M, \omega, \Phi^{\prime}\right)$ is a GKM space. Since $\Phi^{\prime} \circ \pi^{*}=\Phi, \Psi=\Phi^{\xi}=\left(\Phi^{\prime}\right)^{\pi(\xi)}$ is a generic component of the moment map. Note that the vertices and edges of the graphs for the $T$ action and the $T^{\prime}$ action are naturally identical, and that $\pi^{*}$ takes the $T^{\prime}$-weight of each edge to the $T$-weight of the same edge. In particular, $\pi^{*}\left(\left(\Lambda^{\prime}\right)_{\mathrm{p}}^{-}\right)=\Lambda_{\mathrm{p}}^{-}$, where $\left(\Lambda^{\prime}\right)_{\mathrm{p}}^{-}$denotes the $\mathrm{T}^{\prime}$-equivariant Euler class of the negative normal bundle of $\Psi$ at $p$.

Since $T^{\prime}$ acts effectively on $M$, the first part of this proof implies there exists a class $\alpha_{p}^{\prime} \in \mathrm{H}_{\mathrm{T}^{\prime}}^{2 \lambda(p)}(M ; \mathbb{Z})$ satisfying

(1) $\alpha_{p}^{\prime}(p)=\left(\Lambda^{\prime}\right)_{p}^{-}$,

$\left(2^{\prime \prime}\right) \alpha_{p}^{\prime}(q)=0$ for all $q \in M^{\top} \backslash v_{p}$, and

(3) $\alpha_{p}^{\prime}$ is robustly integral at every point $q \in M^{\top}$.

Let $\alpha_{p}:=\Pi_{M}^{*}\left(\alpha_{p}^{\prime}\right) \in H_{T}^{*}(M ; \mathbb{Z})$. Then $\alpha_{p}$ has the desired properties.

\section{Examples}

We conclude our paper with two explicit examples.

Example 5.1. $\mathrm{Fl}\left(\mathbb{C}^{n}\right)$. The standard action of $T=\left(S^{1}\right)^{n}$ induces an action on the complete flag manifold $\mathrm{Fl}\left(\mathbb{C}^{n}\right)$ whose fixed points are given by flags in the coordinate lines $\mathbb{C}^{n}$. These are indexed by permutations $\sigma \in S_{n}$ on $n$ letters; the fixed point corresponding to $\sigma$ is given by

$$
\langle 0\rangle \subset\left\langle f_{\sigma(1)}\right\rangle \subset\left\langle f_{\sigma(1)}, f_{\sigma(2)}\right\rangle \subset \cdots \subset\left\langle f_{\sigma(1)}, f_{\sigma(2)}, \cdots f_{\sigma(n)}\right\rangle=\mathbb{C}^{n},
$$

\footnotetext{
${ }^{6}$ To see this, let ET and $E T^{\prime}$ be contractible spaces on which $T$ and $T^{\prime}$, respectively, act freely. Then $\mathrm{T}$ acts on $\mathrm{ET} \times E T^{\prime}$ by $\mathrm{t} \cdot\left(e, e^{\prime}\right)=\left(\mathrm{t} \cdot \mathrm{e}, \pi(\mathrm{t}) \cdot e^{\prime}\right)$. The projection from $M \times\left(E T \times E T^{\prime}\right)$ to $M \times E T^{\prime}$ descends to a map from $M \times_{T}\left(E T \times E T^{\prime}\right)$ to $M \times_{T^{\prime}} E^{\prime} T^{\prime}$. This induces a map from $\mathrm{H}^{*}\left(M \times_{\mathrm{T}}, \mathrm{ET}^{\prime}\right)=\mathrm{H}_{\mathrm{T}}^{*}(\mathrm{M})$ to $\mathrm{H}^{*}\left(\mathrm{M} \times_{\mathrm{T}}\left(\mathrm{ET} \times \mathrm{ET}^{\prime}\right)\right)=\mathrm{H}_{\mathrm{T}}^{*}(M)$.
} 
where the brackets indicate the span of the vectors and $f_{1}, \ldots, f_{n}$ is the standard basis of $\mathbb{C}^{\mathrm{n}}$. By abuse of notation, we will denote both the permutation and the corresponding fixed point by $\sigma$. Let $\ell(\sigma)$ denote the length of $\sigma$, and choose a generic $\xi$ such that $\ell(\sigma)<\ell\left(\sigma^{\prime}\right)$ implies $\Psi(\sigma)<\Psi\left(\sigma^{\prime}\right)$ for all $\sigma, \sigma^{\prime} \in S_{n}$. Note that $\left(\sigma, \sigma^{\prime}\right) \in E$ if there exists a transposition $t$ such that t $\sigma=\sigma^{\prime}$ and $\ell(\sigma)=\ell\left(\sigma^{\prime}\right)-1$ (in contrast, $\mathrm{E}_{\mathrm{GKM}}$ consists of all $\left(\sigma, \sigma^{\prime}\right)$ such that $t \sigma=\sigma^{\prime}$ ) for some $t$ ). If $t=t_{i j}$ is the transposition switching $i$ and $j$ with $i<j$, then $\eta\left(\sigma, \sigma^{\prime}\right)=x_{i}-x_{j}$ and by our convention, this is considered a positive weight.

We begin by showing that for all edges $\left(\sigma, \sigma^{\prime}\right) \in \mathrm{E}, \Theta\left(\sigma, \sigma^{\prime}\right)=1$. Recall that the weights that occur in the representation of $\mathrm{T}$ on the negative normal bundle at a point $\sigma$ are positive weights. The length $\ell(\sigma)$ is also the number of positive weights at $\sigma$. The weight $\eta \neq \eta\left(\sigma, \sigma^{\prime}\right)$ is a positive weight at $\sigma^{\prime}$ if and only if $t_{i j} \eta$ is a positive weight $\sigma$. Thus there is a bijection of positive weights at $\sigma$ and positive weights at $\sigma^{\prime}$ excepting $\eta\left(\sigma^{\prime}, \sigma\right)$. Moreover, for each weight $\eta$ at $\sigma$, the weight $t_{i j} \eta$ at $\sigma^{\prime}$ has the property that $\eta=t_{i j} \eta$ $\bmod \eta\left(\sigma, \sigma^{\prime}\right)$. It follows that $\Theta_{\eta\left(\sigma, \sigma^{\prime}\right)}=1$.

Now consider any fixed point $\sigma$, and let $\alpha_{\sigma}$ be the associated canonical class. Theorem 1.6 says for any $\mu \in M^{\top}$,

$$
\alpha_{\sigma}(\mu)=\Lambda_{\mu}^{-} \sum_{\mathbf{r} \in \Sigma_{\sigma}^{\mu}} \prod_{i=1}^{|r|} \frac{\Phi\left(r_{i}\right)-\Phi\left(r_{i-1}\right)}{\Phi(\mu)-\Phi\left(r_{i-1}\right)} \frac{1}{\eta\left(r_{i-1}, r_{i}\right)} .
$$

Note that $\eta\left(r_{i-1}, r_{i}\right)$ is positive. Similarly, $\Lambda_{\mu}^{-}$is a product of positive weights. Finally, $\Phi\left(r_{i}\right)-\Phi\left(r_{i-1}\right)$ and $\Phi(\mu)-\Phi\left(r_{i-1}\right)$ are positive for each i. More precisely, $\Phi\left(r_{i}\right)-\Phi\left(r_{i-1}\right)=k_{i} \eta\left(r_{i-1}, r_{i}\right)$ where $k_{i} \in \mathbb{Q}^{+}$and $\Phi(\mu)-\Phi\left(r_{i-1}\right)=\sum_{j=1}^{m} k_{j} \eta\left(s_{j-1}, s_{j}\right)$, where $j$ indexes the vertices in a path $\left(s_{0}, \ldots, s_{m}\right)$ from $s_{0}=r_{i-1}$ to $s_{m}=\mu$. Thus every term in expression (5.1) is positive.

Example 5.2. The integer $\Theta(p, q)$ is not always positive; when it is negative, the canonical class $\alpha_{p}$ restricts to a negative value at $q$. In [To1], the second author demonstrated the existence of a GKM space that does not have a T-invariant Kähler metric. The corresponding GKM graph $\mathrm{E}_{\mathrm{GKM}}$ can be expressed as the image of the singular set under the moment map $\Phi$, pictured in Figure 1, where we have represented each pair of edges $(p, q)$ and $(q, p)$ by one drawn edge. Let $\xi \in \mathfrak{t}$ be as indicated, and note that $\Psi=\Phi^{\xi}$ is an index increasing component of the moment map. Consider the edge corresponding to $(p, q)$. Indicated on the figure are the positive weights (excluding $\eta(p, q)$ ) of the $T$ action on the negative normal bundles at $p$ and $q$, according to the choice of $\xi$ indicated. Under the map $\rho_{\eta(p, q)}$, these vectors project to vectors opposite in sign (and of equal magnitude). Thus $\Theta(p, q)<0$. It immediately follows from Theorem 1.6 that $\alpha_{p}(q)<0$. 


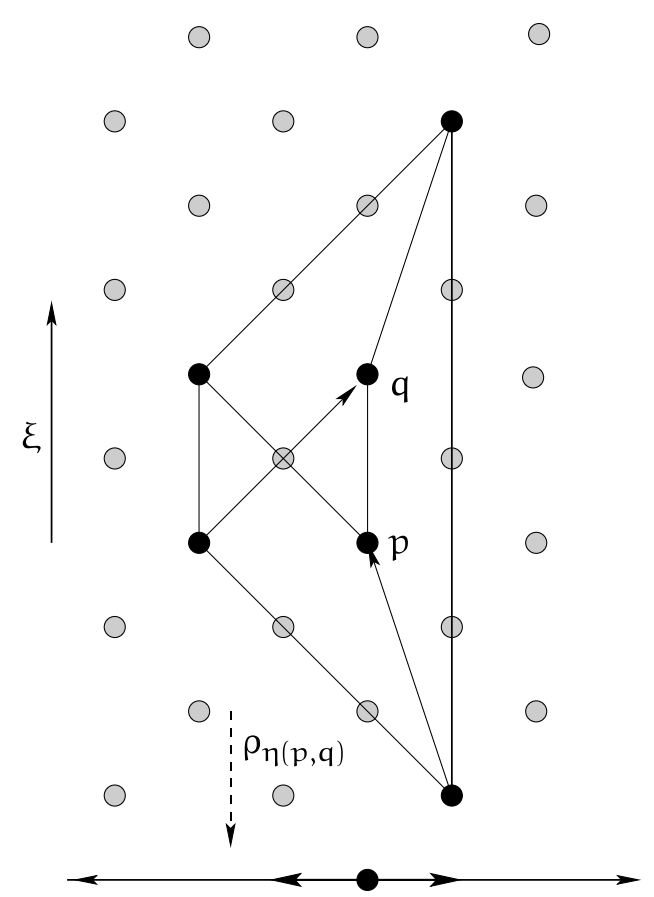

Figure 1. A non-Kähler GKM space. The restriction of the canonical class $\alpha_{p}$ to $q$ is negative.

\section{References}

[BGG] I. Bernstein, I. Gelfand and S. Gelfand, Schubert cells, and the cohomolooy of the spaces G/P, Uspehi Mat. Nauk 28(3) (1973), 3-26.

[Bi] S. Billey, Kostant polynomials and the cohomology ring for G/B, Duke J. Math. 96(1) (1998), 205-224.

[BT] R. Bott and L. Tu, Differential forms in algebraic topology, Graduate Texts in Mathematics, 82, Springer-Verlag, New York, 1982.

[GGK] V. L. Ginzburg, V. Guillemin and Y. Karshon, Moment maps, cobordisms, and Hamiltonian group actions, Math. Surveys and Monographs, 98, American Mathematical Society, 2002.

[GKM] M. Goresky, R. Kottwitz and R. MacPherson, Equivariant cohomology, Koszul duality, and the localization theorem. Invent. Math. 131(1) (1998), 25-83.

[GZ] V. Guillemin and C. Zara, Combinatorial formulas for products of Thom classes, Geometry, Mechanics, and Dynamics, Springer, New York, 2002, 363-405.

[Ki] F. Kirwan, Cohomology of quotients in symplectic and algebraic geometry. Mathematical Notes, Princeton University Press, 1984.

[Kn99] A. Knutson, A Littelmann-type formula for Duistermaat-Heckman measures, Invent. Math. 135(1) (1999), 185-200.

[Kn10] A. Knutson, A compactly supported formula for equivariant localization and simplicial complexes of Biatynicki-Birula decompositions, to appear in Pure Appl. Math. Q. special issue in honor of M. Atiyah. 
[MS] D. McDuff and D. Salamon, J-holomorphic curves and symplectic topology, 52, American Mathematical Society Colloquium Publications, Providence, RI, 2004.

[MT] D. McDuff and S. Tolman, Topological properties of Hamiltonian circle actions, IMRP Int. Math. Res. Pap. 72826 (2006), 1-77.

[ST] S. Sabatini and S. Tolman, A simpler path formula for equivariant cohomology, in preparation.

[To1] S. Tolman, Examples of non-Kähler Hamiltonian torus actions, Invent. Math. 131(2) (1998), 299-310.

[To2] S. Tolman, On a symplectic generalization of Petrie's conjecture, accepted by Trans. Am. Math. Soc. (2008).

[TW] S. Tolman and J. Weitsman, On the cohomology rings of Hamiltonian T-spaces, Northern California Symplectic Geometry Seminar, American Mathematical Society Translation Series 2, 196, American Mathematical Society, Providence, RI, 1999, 251-258.

[Za] C. Zara, Positivity of equivariant Schubert classes through moment map degeneration, preprint, arXiv:0904.0902v1.

Mathematical Sciences

George Mason University

MS 3F2, 4400 UNIVERSITY DR.

FAIRFAX, VA 22030, USA

E-mail address: rgoldin@math.gmu.edu

Department of Mathematics

UNIVERSITY OF ILLINOIS URBANA-CHAMPAIGN

1409 W. Green Street

URBANA, IL 61801, USA

E-mail address: stolman@math.uiuc.edu

Received 07/28/2008, accepted 12/10/2008

First author was partially supported by NSF-DMS Grant \#0606869. Second author was partially supported by NSF-DMS Grant \#0707122. 
